TRANSACTIONS OF THE

AMERICAN MATHEMATICAL SOCIETY

Volume 358, Number 11, November 2006, Pages 5139-5159

S 0002-9947(06)03911-0

Article electronically published on June 15, 2006

\title{
JOINTLY HYPONORMAL PAIRS OF COMMUTING SUBNORMAL OPERATORS NEED NOT BE JOINTLY SUBNORMAL
}

\author{
RAÚL E. CURTO AND JASANG YOON
}

\begin{abstract}
We construct three different families of commuting pairs of subnormal operators, jointly hyponormal but not admitting commuting normal extensions. Each such family can be used to answer in the negative a 1988 conjecture of R. Curto, P. Muhly and J. Xia. We also obtain a sufficient condition under which joint hyponormality does imply joint subnormality.
\end{abstract}

\section{INTRODUCTION}

Let $\mathcal{H}$ be a complex Hilbert space and let $\mathcal{B}(\mathcal{H})$ denote the algebra of bounded linear operators on $\mathcal{H}$. For $S, T \in \mathcal{B}(\mathcal{H})$ let $[S, T]:=S T-T S$. We say that an $n$-tuple $\mathbf{T}=\left(T_{1}, \cdots, T_{n}\right)$ of operators on $\mathcal{H}$ is (jointly) hyponormal if the operator matrix

$$
\left[\mathbf{T}^{*}, \mathbf{T}\right]:=\left(\begin{array}{cccc}
{\left[T_{1}^{*}, T_{1}\right]} & {\left[T_{2}^{*}, T_{1}\right]} & \cdots & {\left[T_{n}^{*}, T_{1}\right]} \\
{\left[T_{1}^{*}, T_{2}\right]} & {\left[T_{2}^{*}, T_{2}\right]} & \cdots & {\left[T_{n}^{*}, T_{2}\right]} \\
\vdots & \vdots & \ldots & \vdots \\
{\left[T_{1}^{*}, T_{n}\right]} & {\left[T_{2}^{*}, T_{n}\right]} & \cdots & {\left[T_{n}^{*}, T_{n}\right]}
\end{array}\right)
$$

is positive on the direct sum of $n$ copies of $\mathcal{H}$ (cf. [Ath], [CMX]). The $n$-tuple $\mathbf{T}$ is said to be normal if $\mathbf{T}$ is commuting and each $T_{i}$ is normal, and $\mathbf{T}$ is subnormal if $\mathbf{T}$ is the restriction of a normal $n$-tuple to a common invariant subspace. Clearly, normal $\Rightarrow$ subnormal $\Rightarrow$ hyponormal. The Bram-Halmos criterion states that an operator $T \in \mathcal{B}(\mathcal{H})$ is subnormal if and only if the $k$-tuple $\left(T, T^{2}, \cdots, T^{k}\right)$ is hyponormal for all $k \geq 1$.

For $\alpha \equiv\left\{\alpha_{n}\right\}_{n=0}^{\infty}$ a bounded sequence of positive real numbers (called weights), let $W_{\alpha}: \ell^{2}\left(\mathbb{Z}_{+}\right) \rightarrow \ell^{2}\left(\mathbb{Z}_{+}\right)$be the associated unilateral weighted shift, defined by $W_{\alpha} e_{n}:=\alpha_{n} e_{n+1}$ (all $n \geq 0$ ), where $\left\{e_{n}\right\}_{n=0}^{\infty}$ is the canonical orthonormal basis in $\ell^{2}\left(\mathbb{Z}_{+}\right)$. The moments of $\alpha$ are given as

$$
\gamma_{k} \equiv \gamma_{k}(\alpha):=\left\{\begin{array}{cl}
1 & \text { if } k=0 \\
\alpha_{0}^{2} \cdot \ldots \cdot \alpha_{k-1}^{2} & \text { if } k>0
\end{array}\right.
$$

It is easy to see that $W_{\alpha}$ is never normal, and that it is hyponormal if and only if $\alpha_{0} \leq \alpha_{1} \leq \cdots$. Similarly, consider double-indexed positive bounded sequences $\alpha_{\mathbf{k}}, \beta_{\mathbf{k}} \in \ell^{\infty}\left(\mathbb{Z}_{+}^{2}\right), \mathbf{k} \equiv\left(k_{1}, k_{2}\right) \in \mathbb{Z}_{+}^{2}:=\mathbb{Z}_{+} \times \mathbb{Z}_{+}$, and let $\ell^{2}\left(\mathbb{Z}_{+}^{2}\right)$ be the Hilbert

Received by the editors January 22, 2004 and, in revised form, December 5, 2004.

2000 Mathematics Subject Classification. Primary 47B20, 47B37, 47A13, 28A50; Secondary 44A60, 47-04, 47A20.

Key words and phrases. Jointly hyponormal pairs, subnormal pairs, 2-variable weighted shifts. This research was partially supported by NSF Grant DMS-0099357. 
space of square-summable complex sequences indexed by $\mathbb{Z}_{+}^{2}$. (Recall that $\ell^{2}\left(\mathbb{Z}_{+}^{2}\right)$ is canonically isometrically isomorphic to $\ell^{2}\left(\mathbb{Z}_{+}\right) \otimes \ell^{2}\left(\mathbb{Z}_{+}\right)$.) We define the 2-variable weighted shift $\mathbf{T} \equiv\left(T_{1}, T_{2}\right)$ by

$$
\begin{aligned}
& T_{1} e_{\mathbf{k}}:=\alpha_{\mathbf{k}} e_{\mathbf{k}+\varepsilon_{1}}, \\
& T_{2} e_{\mathbf{k}}:=\beta_{\mathbf{k}} e_{\mathbf{k}+\varepsilon_{2}},
\end{aligned}
$$

where $\varepsilon_{1}:=(1,0)$ and $\varepsilon_{2}:=(0,1)$. Clearly,

$$
T_{1} T_{2}=T_{2} T_{1} \Longleftrightarrow \beta_{\mathbf{k}+\varepsilon_{1}} \alpha_{\mathbf{k}}=\alpha_{\mathbf{k}+\varepsilon_{2}} \beta_{\mathbf{k}} \text { (all } \mathbf{k} \text { ). }
$$

In an entirely similar way one can define multivariable weighted shifts. Trivially, a pair of unilateral weighted shifts $W_{\alpha}$ and $W_{\beta}$ gives rise to a 2-variable weighted shift $\mathbf{T} \equiv\left(T_{1}, T_{2}\right)$, if we let $\alpha_{\left(k_{1}, k_{2}\right)}:=\alpha_{k_{1}}$ and $\beta_{\left(k_{1}, k_{2}\right)}:=\beta_{k_{2}}\left(\right.$ all $\left.k_{1}, k_{2} \in \mathbb{Z}_{+}\right)$. In this case, $\mathbf{T}$ is subnormal (resp. hyponormal) if and only if so are $T_{1}$ and $T_{2}$. In fact, under the canonical identification of $\ell^{2}\left(\mathbb{Z}_{+}^{2}\right)$ and $\ell^{2}\left(\mathbb{Z}_{+}\right) \otimes \ell^{2}\left(\mathbb{Z}_{+}\right), T_{1} \cong I \otimes W_{\alpha}$ and $T_{2} \cong W_{\beta} \otimes I$, and $\mathbf{T}$ is also doubly commuting. For this reason, we do not focus our attention on shifts of this type, but use them only when the above-mentioned triviality is desirable or needed.

We now recall a well-known characterization of subnormality for single variable weighted shifts, due to C. Berger (cf. [Con, III.8.16]): $W_{\alpha}$ is subnormal if and only if there exists a probability measure $\xi$ supported in $\left[0,\left\|W_{\alpha}\right\|^{2}\right]$ (called the Berger measure of $\left.W_{\alpha}\right)$ such that $\gamma_{k}(\alpha):=\alpha_{0}^{2} \cdot \ldots \cdot \alpha_{k-1}^{2}=\int t^{k} d \xi(t) \quad(k \geq 1)$. If $W_{\alpha}$ is subnormal, and if for $h \geq 1$ we let $\mathcal{M}_{h}:=\bigvee\left\{e_{n}: n \geq h\right\}$ denote the invariant subspace obtained by removing the first $h$ vectors in the canonical orthonormal basis of $\ell^{2}\left(\mathbb{Z}_{+}\right)$, then the Berger measure of $\left.W_{\alpha}\right|_{\mathcal{M}_{h}}$ is $\frac{1}{\gamma_{h}} t^{h} d \xi(t)$.

An important class of subnormal weighted shifts is obtained by considering measures $\mu$ with exactly two atoms $t_{0}$ and $t_{1}$. These shifts arise naturally in the Subnormal Completion Problem $\mathrm{CuFi} 3$ and in the theory of truncated moment problems (cf. CuFi1, CuFi4). For $t_{0}, t_{1} \in \mathbb{R}_{+}$with $t_{0}<t_{1}$, and $\rho_{0}, \rho_{1}>0$ with $\rho_{0}+\rho_{1}=1$, the moments of the 2 -atomic probability measure $\xi:=\rho_{0} \delta_{t_{0}}+\rho_{1} \delta_{t_{1}}$ (here $\delta_{p}$ denotes the point-mass probability measure with support the singleton $\{p\}$ ) satisfy the 2-step recursive relation $\gamma_{n+2}=\varphi_{0} \gamma_{n}+\varphi_{1} \gamma_{n+1}(n \geq 0)$; at the weight level, this can be written as $\alpha_{n+1}^{2}=\frac{\varphi_{0}}{\alpha_{n}^{2}}+\varphi_{1}(n \geq 0)$. More generally, any finitely atomic Berger measure corresponds to a recursively generated weighted shift (i.e., one whose moments satisfy an $r$-step recursive relation); in fact, $r=$ card supp $\xi$. In the special case of $r=2$, the theory of recursively generated weighted shifts makes contact with the work of J. Stampfli in [Sta], in which he proved that given three positive numbers $\alpha_{0}<\alpha_{1}<\alpha_{2}$, it is always possible to find a subnormal weighted shift, denoted $W_{\left(\alpha_{0}, \alpha_{1}, \alpha_{2}\right)^{\wedge}}$, whose first three weights are $\alpha_{0}, \alpha_{1}$ and $\alpha_{2}$. In this case, the coefficients of recursion (cf. [CuFi2, Example 3.12], CuFi3, Section 3], Cu3, Section 1, p. 81]) are given by

$$
\varphi_{0}=-\frac{\alpha_{0}^{2} \alpha_{1}^{2}\left(\alpha_{2}^{2}-\alpha_{1}^{2}\right)}{\alpha_{1}^{2}-\alpha_{0}^{2}} \text { and } \varphi_{1}=\frac{\alpha_{1}^{2}\left(\alpha_{2}^{2}-\alpha_{0}^{2}\right)}{\alpha_{1}^{2}-\alpha_{0}^{2}},
$$

the atoms $t_{0}$ and $t_{1}$ are the roots of the equation

$$
t^{2}-\left(\varphi_{0}+\varphi_{1} t\right)=0
$$

and the densities $\rho_{0}$ and $\rho_{1}$ uniquely solve the $2 \times 2$ system of equations

$$
\left\{\begin{array}{ccc}
\rho_{0}+\rho_{1} & = & 1 \\
\rho_{0} t_{0}+\rho_{1} t_{1} & = & \alpha_{0}^{2}
\end{array}\right.
$$


We also recall the notion of moment of order $\mathbf{k}$ for a pair $(\alpha, \beta)$ satisfying (1.1). Given $\mathbf{k} \in \mathbb{Z}_{+}^{2}$, the moment of $(\alpha, \beta)$ of order $\mathbf{k}$ is

$$
\begin{aligned}
& \gamma_{\mathbf{k}} \equiv \gamma_{\mathbf{k}}(\alpha, \beta) \\
& :=\left\{\begin{array}{cc}
1 & \text { if } \mathbf{k}=0 \\
\alpha_{(0,0)}^{2} \cdot \ldots \cdot \alpha_{\left(k_{1}-1,0\right)}^{2} & \text { if } k_{1} \geq 1 \text { and } k_{2}=0 \\
\beta_{(0,0)}^{2} \cdot \ldots \cdot \beta_{\left(0, k_{2}-1\right)}^{2} & \text { if } k_{1}=0 \text { and } k_{2} \geq 1 \\
\alpha_{(0,0)}^{2} \cdot \ldots \cdot \alpha_{\left(k_{1}-1,0\right)}^{2} \cdot \beta_{\left(k_{1}, 0\right)}^{2} \cdot \ldots \cdot \beta_{\left(k_{1}, k_{2}-1\right)}^{2} & \text { if } k_{1} \geq 1 \text { and } k_{2} \geq 1 .
\end{array}\right.
\end{aligned}
$$

We remark that, due to the commutativity condition (1.1), $\gamma_{\mathbf{k}}$ can be computed using any nondecreasing path from $(0,0)$ to $\left(k_{1}, k_{2}\right)$.

Theorem 1.1 (Berger's Theorem, 2-variable case) (JeLu]). A 2-variable weighted shift $\mathbf{T} \equiv\left(T_{1}, T_{2}\right)$ admits a commuting normal extension if and only if there is a probability measure $\mu$ defined on the 2-dimensional rectangle $R=\left[0, a_{1}\right] \times\left[0, a_{2}\right]$ $\left(a_{i}:=\left\|T_{i}\right\|^{2}\right)$ such that $\gamma_{\mathbf{k}}=\iint_{R} \mathbf{t}^{\mathbf{k}} d \mu(\mathbf{t}):=\iint_{R} t_{1}^{k_{1}} t_{2}^{k_{2}} d \mu\left(t_{1}, t_{2}\right) \quad\left(\right.$ all $\left.\mathbf{k} \in \mathbb{Z}_{+}^{2}\right)$.

Clearly, each component $T_{i}$ of a subnormal 2 -variable weighted shift $\mathbf{T} \equiv\left(T_{1}, T_{2}\right)$ must be subnormal. For instance, $T_{1} \cong \bigoplus_{j=0}^{\infty} W_{\alpha^{(j)}}$, where $\alpha_{i}^{(j)}:=\alpha_{(i, j)}$, so that $W_{\alpha^{(j)}}$ has Berger measure $d \nu_{j}\left(t_{1}\right):=\frac{1}{\gamma_{(0, j)}} \int_{\left[0, a_{2}\right]} t_{2}^{j} d \Phi_{t_{1}}\left(t_{2}\right)$, where $d \mu\left(t_{1}, t_{2}\right) \equiv$ $d \Phi_{t_{1}}\left(t_{2}\right) d \eta\left(t_{1}\right)$ is the canonical disintegration of $\mu$ by horizontal slices. On the other hand, if we only know that each of $T_{1}, T_{2}$ is subnormal, and that they commute, the following problem is natural.

Problem 1.2 (Lifting Problem for Commuting Subnormals). Find necessary and sufficient conditions on $T_{1}$ and $T_{2}$ to guarantee the subnormality of $\mathbf{T} \equiv\left(T_{1}, T_{2}\right)$.

It is well known that the above-mentioned necessary conditions do not suffice (cf. $\mathrm{Cu} 1]$ ). In terms of the marginal measures, the problem can be phrased as a reconstruction-of-measure problem, that is, under what conditions on the single variable measures $\left\{\nu_{j}\right\}_{j=0}^{\infty}$ and $\left\{\omega_{i}\right\}_{i=0}^{\infty}$ associated with $T_{1}$ and $T_{2}$, respectively, does there exist a 2 -variable measure $\mu$ correctly interpolating all the powers $t_{1}^{k_{1}} t_{2}^{k_{2}}\left(k_{1}, k_{2} \geq 0\right) ?$

To detect hyponormality for 2 -variable weighted shifts, there is a simple criterion involving a base point $\mathbf{k}$ in $\mathbb{Z}_{+}^{2}$ and its five neighboring points in $\mathbf{k}+\mathbb{Z}_{+}^{2}$ at path distance at most 2 (cf. Figure 11).

Theorem 1.3 (Six-point Test) ([Cu1]). Let $\mathbf{T} \equiv\left(T_{1}, T_{2}\right)$ be a 2 -variable weighted shift, with weight sequences $\alpha$ and $\beta$. Then

$$
\begin{gathered}
{\left[\mathbf{T}^{*}, \mathbf{T}\right] \geq 0 \Leftrightarrow\left(\left(\left[T_{j}^{*}, T_{i}\right] e_{\mathbf{k}+\varepsilon_{j}}, e_{\mathbf{k}+\varepsilon_{i}}\right)\right)_{i, j=1}^{2} \geq 0\left(\text { all } \mathbf{k} \in \mathbf{Z}_{+}^{2}\right)} \\
\Leftrightarrow\left(\begin{array}{cc}
\alpha_{\mathbf{k}+\varepsilon_{1}}^{2}-\alpha_{\mathbf{k}}^{2} & \alpha_{\mathbf{k}+\varepsilon_{2}} \beta_{\mathbf{k}+\varepsilon_{1}}-\alpha_{\mathbf{k}} \beta_{\mathbf{k}} \\
\alpha_{\mathbf{k}+\varepsilon_{2}} \beta_{\mathbf{k}+\varepsilon_{1}}-\alpha_{\mathbf{k}} \beta_{\mathbf{k}} & \beta_{\mathbf{k}+\varepsilon_{2}}^{2}-\beta_{\mathbf{k}}^{2}
\end{array}\right) \geq 0\left(\text { all } \mathbf{k} \in \mathbf{Z}_{+}^{2}\right) .
\end{gathered}
$$

Unlike the single variable case, in which there is a clear separation between hyponormality and subnormality (cf. CuFi3, Cu3], CuLe), much less is known about the multivariable case. In this paper we will construct three conceptually different families of counterexamples to the following conjecture.

Conjecture 1.4 ([CMX]). Let $\mathbf{T} \equiv\left(T_{1}, T_{2}\right)$ be a pair of commuting subnormal operators on $\mathcal{H}$. Then $\mathbf{T}$ is subnormal if and only if $\mathbf{T}$ is hyponormal. 


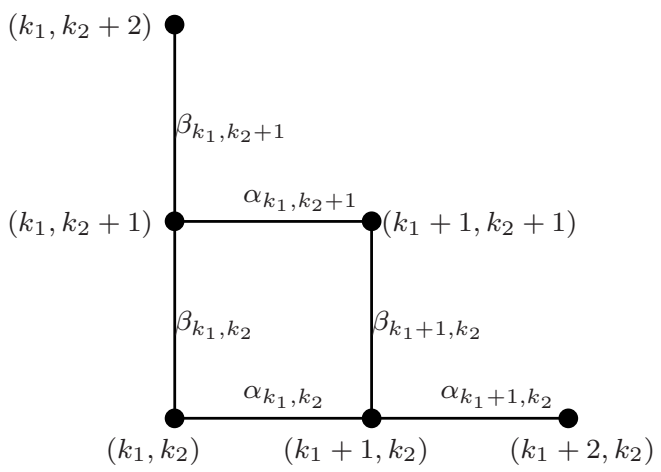

Figure 1. Weight diagram used in the Six-point Test

We mention that M. Dritschel and S. McCullough, working independently, have been able to obtain a separate example $([\overline{\mathrm{DrMcC}}])$. We shall see in Section 4 that their example is a special case of a general construction that produces nonsubnormal hyponormal pairs with $T_{1} \cong T_{2}$.

We now formulate an improved version of a result due to $\mathrm{R}$. Curto.

Proposition 1.5 (Subnormal backward extension of a 1-variable weighted shift) (cf. [Cu2]). Let $T$ be a weighted shift whose restriction $T_{\mathcal{M}}:=\left.T\right|_{\mathcal{M}}$ to $\mathcal{M}:=$ $\bigvee\left\{e_{1}, e_{2}, \cdots\right\}$ is subnormal, with Berger measure $\mu_{\mathcal{M}}$. Then $T$ is subnormal (with associated measure $\mu$ ) if and only if

(i) $\frac{1}{t} \in L^{1}\left(\mu_{\mathcal{M}}\right)$,

(ii) $\alpha_{0}^{2} \leq\left(\left\|\frac{1}{t}\right\|_{L^{1}\left(\mu_{\mathcal{M}}\right)}\right)^{-1}$.

In this case, $d \mu(t)=\frac{\alpha_{0}^{2}}{t} d \mu_{\mathcal{M}}(t)+\left(1-\alpha_{0}^{2}\left\|\frac{1}{t}\right\|_{L^{1}\left(\mu_{\mathcal{M}}\right)}\right) d \delta_{0}(t)$, where $\delta_{0}$ denotes Dirac measure at 0 . In particular, $T$ is never subnormal when $\mu_{\mathcal{M}}(\{0\})>0$.

Proof. $\Rightarrow$ ) We first observe that the moments of $T$ and $T_{\mathcal{M}}$ are related by the equation

$$
\gamma_{k}\left(T_{\mathcal{M}}\right) \equiv \alpha_{1}^{2} \cdots \alpha_{k}^{2}=\frac{\gamma_{k+1}(T)}{\alpha_{0}^{2}}
$$

so that

$$
\frac{1}{\alpha_{0}^{2}} \int t^{k+1} d \mu(t)=\int t^{k} d \mu_{\mathcal{M}}(t) \quad(\text { all } k \geq 0),
$$

that is, $t d \mu(t)=\alpha_{0}^{2} d \mu_{\mathcal{M}}(t)$. It follows at once that

$$
d \mu(t)=\lambda d \delta_{0}(t)+\frac{\alpha_{0}^{2}}{t} d \mu_{\mathcal{M}}(s),
$$

where $\lambda \geq 0$. Since $\int d \mu=1$, we must have $\frac{1}{t} \in L^{1}\left(\mu_{\mathcal{M}}\right)$ and $\alpha_{0}^{2}\left\|\frac{1}{t}\right\|_{L^{1}\left(\mu_{\mathcal{M}}\right)} \leq 1$. Finally, it is straightforward to verify that $\lambda=1-\alpha_{0}^{2}\left\|\frac{1}{t}\right\|_{L^{1}\left(\mu_{\mathcal{M}}\right)}$.

$\Leftarrow)$ Let

$$
d \mu(t):=\frac{\alpha_{0}^{2}}{t} d \mu_{\mathcal{M}}(t)+\left(1-\alpha_{0}^{2}\left\|\frac{1}{t}\right\|_{L^{1}\left(\mu_{\mathcal{M}}\right)}\right) d \delta_{0}(t) .
$$


By hypotheses, $\mu$ is a positive Borel measure on $\left[0,\|T\|^{2}\right]$. Moreover,

$$
\int d \mu=\alpha_{0}^{2} \int \frac{1}{t} d \mu_{\mathcal{M}}+\left(1-\alpha_{0}^{2}\left\|\frac{1}{t}\right\|_{L^{1}\left(\mu_{\mathcal{M}}\right)}\right) \int d \delta_{0}=1,
$$

and for $k \geq 1$,

$$
\begin{aligned}
\int t^{k} d \mu(t) & =\alpha_{0}^{2} \int t^{k} \frac{1}{t} d \mu_{\mathcal{M}}(t)+\left(1-\alpha_{0}^{2}\left\|\frac{1}{t}\right\|_{L^{1}\left(\mu_{\mathcal{M}}\right)}\right) \int t^{k} d \delta_{0}(t) \\
& =\alpha_{0}^{2} \int t^{k-1} d \mu_{\mathcal{M}}(t)=\alpha_{0}^{2} \gamma_{k-1}\left(T_{\mathcal{M}}\right)=\gamma_{k}(T) .
\end{aligned}
$$

Therefore, $T$ is subnormal, with Berger measure $\mu$.

Notation 1.6. The maximum possible value for $\alpha_{0}$ in Proposition 1.5. namely $\left(\left\|\frac{1}{t}\right\|_{L^{1}\left(\mu_{\mathcal{M}}\right)}\right)^{-1}$, will be denoted by

$$
\alpha_{e x t} \equiv \alpha_{e x t}\left(\mu_{\mathcal{M}}\right)
$$

Observe that shift $\left(\alpha_{\text {ext }}, \alpha_{1}, \alpha_{2}, \cdots\right)$ is subnormal, with Berger measure $d \mu(t)=$ $\frac{\alpha_{0}^{2}}{t} d \mu_{\mathcal{M}}(t)$. For example, if $B_{+}$denotes the Bergman shift on $\ell^{2}\left(\mathbb{Z}_{+}\right)$, then $\left.B_{+}\right|_{\mathcal{M}}$ is subnormal, with Berger measure $d \mu(t):=2 t d t$ on $[0,1]$. Then $d \mu_{e x t}(t)=d t$, so in this case the extremal measure $\mu_{\text {ext }}$ is the Berger measure of $B_{+}$.

More generally, given a (1-variable) subnormal weighted shift $W_{\eta}$ with weight sequence $\eta_{1} \leq \eta_{2} \leq \cdots$ and Berger measure $\nu$, we let

$$
\eta_{\text {ext }}:=\left\{\begin{array}{cc}
0 & \text { if } \frac{1}{t} \notin L^{1}(\nu), \\
\left(\left\|\frac{1}{t}\right\|_{L^{1}(\nu)}\right)^{-1} & \text { if } \frac{1}{t} \in L^{1}(\nu) .
\end{array}\right.
$$

Observe that when the weight sequence $\eta$ is strictly increasing and $\frac{1}{t} \in L^{1}(\nu)$, we must necessarily have

$$
\eta_{\text {ext }}<\eta_{1}
$$

by [Sta, Theorem 6]. On occasion, we will write $\operatorname{shift}\left(\alpha_{0}, \alpha_{1}, \cdots\right)$ to denote the weighted shift with weight sequence $\left\{\alpha_{k}\right\}_{k=0}^{\infty}$. We also denote by $U_{+}:=$ $\operatorname{shift}(1,1, \cdots)$ the (unweighted) unilateral shift, and for $0<a<1$ we let $S_{a}:=$ $\operatorname{shift}(a, 1,1, \cdots)$. Observe that the Berger measures of $U_{+}$and $S_{a}$ are $\delta_{1}$ and $\left(1-a^{2}\right) \delta_{0}+a^{2} \delta_{1}$, respectively, where $\delta_{p}$ denotes the point-mass probability measure with support the singleton $\{p\}$. Finally, we let $B_{+}$denote the Bergman shift, whose Berger measure is Lebesgue measure on the interval [0,1]; the weights of $B_{+}$are given by the formula $\alpha_{n}:=\sqrt{\frac{n+1}{n+2}}(n \geq 0)$.

We conclude this section with a result that will be needed in Section 3

Lemma 1.7 (cf. CuFi3, Theorem 3.10]). For $0<\alpha_{0}<\alpha_{1}<\alpha_{2}$, let $W_{\left(\alpha_{0}, \alpha_{1}, \alpha_{2}\right)^{\text {) }}}$ be the weighted shift described by (1.2), (1.3) and (1.4). Now consider $W_{\eta}:=$ $\operatorname{shift}\left(\alpha_{1}, \alpha_{2}, \cdots\right)$, that is, $W_{\eta}$ is the restriction of $W_{\left(\alpha_{0}, \alpha_{1}, \alpha_{2}\right)}$, to $\mathcal{M}$. Then $\eta_{\text {ext }}=$ $\alpha_{0}$. 


\section{The FIRST FAMILY OF COUNTEREXAMPLES}

Recall that a unilateral weighted shift $W_{\alpha}$ is subnormal if and only if there exists a probability measure $\xi \equiv \xi_{\alpha}$ supported in $\left[0,\left\|W_{\alpha}\right\|^{2}\right]$ such that $\gamma_{k}(\alpha):=$ $\alpha_{0}^{2} \cdot \ldots \cdot \alpha_{k-1}^{2}=\int t^{k} d \xi(t)(k \geq 1)$. For instance, when $\alpha_{1}=\alpha_{2}=\cdots=1$ (i.e., $\left.W_{\alpha} \equiv \operatorname{shift}\left(\alpha_{0}, 1,1, \cdots\right)\right)$, we have $\xi_{\alpha}=\left(1-\alpha_{0}^{2}\right) \delta_{0}+\alpha_{0}^{2} \delta_{1}$. The proof of the following lemma is straightforward.

Lemma 2.1. Given two 1-variable weight sequences $\alpha$ and $\beta$, the 2-variable weighted shift $\left(I \otimes W_{\alpha}, W_{\beta} \otimes I\right)$ is always subnormal, with Berger measure $\mu:=$ $\xi_{\alpha} \times \xi_{\beta}$.

Definition 2.2. Let $\mu$ and $\nu$ be two positive measures on $\mathbb{R}_{+}$. We say that $\mu \leq \nu$ on $X:=\mathbb{R}_{+}$if $\mu(E) \leq \nu(E)$ for all Borel subset $E \subseteq \mathbb{R}_{+}$; equivalently, $\mu \leq \nu$ if and only if $\int f d \mu \leq \int f d \nu$ for all $f \in C(X)$ such that $f \geq 0$ on $\mathbb{R}_{+}$.

Definition 2.3. Let $\mu$ be a probability measure on $X \times Y \equiv \mathbb{R}_{+} \times \mathbb{R}_{+}$, and assume that $\frac{1}{t} \in L^{1}(\mu)$. The extremal measure $\mu_{\text {ext }}$ (which is also a probability measure) on $X \times Y$ is given by $d \mu_{e x t}(s, t):=\left(1-\delta_{0}(t)\right) \frac{1}{t\left\|\frac{1}{t}\right\|_{L^{1}(\mu)}} d \mu(s, t)$.

Definition 2.4. Given a measure $\mu$ on $X \times Y$, the marginal measure $\mu^{X}$ is given by $\mu^{X}:=\mu \circ \pi_{X}^{-1}$, where $\pi_{X}: X \times Y \rightarrow X$ is the canonical projection onto $X$. Thus, $\mu^{X}(E)=\mu(E \times Y)$, for every $E \subseteq X$. Observe that if $\mu$ is a probability measure, then so is $\mu^{X}$.

Lemma 2.5. Let $\mu$ be the Berger measure of a 2-variable weighted shift $\mathbf{T}$, and let $\nu$ be the Berger measure of shift $\left(\alpha_{00}, \alpha_{10}, \cdots\right)$. Then $\nu=\mu^{X}$. As a consequence, $\iint f(s) d \mu(s, t)=\int f(s) d \mu^{X}(s)$ for all $f \in C(X)$.

Proof. Observe that $\int s^{i} d \nu(s)=\gamma_{i 0}=\iint s^{i} d \mu(s, t)$ for all $i \geq 0$. It follows that $\int f(s) d \nu(s)=\iint f(s) d \mu(s, t)$ for all $f \in C(X)$. Then, for any Borel set $E \subseteq X$, we have

$$
\nu(E)=\int \chi_{E} d \nu=\iint \chi_{E \times Y} d \mu=\mu(E \times Y)=\mu^{X}(E),
$$

as desired. The second assertion follows immediately from what we have established.

Corollary 2.6. Let $\mu$ be the Berger measure of a 2-variable weighted shift $\mathbf{T}$. For $j \geq 1$, let $d \mu_{j}(s, t):=\frac{1}{\gamma_{0 j}} t^{j} d \mu(s, t)$. Then the Berger measure of shift $\left(\alpha_{0 j}, \alpha_{1 j}, \cdots\right)$ is $\nu_{j} \equiv \mu_{j}^{X}$.

Example 2.7. Let $\mu:=\xi \times \eta$ be a probability product measure on $X \times Y$. Then $\mu^{X}=\xi$.

Lemma 2.8. Let $\mu$ and $\omega$ be two measures on $X \times Y$, and assume that $\mu \leq \omega$. Then $\mu^{X} \leq \omega^{X}$.

Proof. Straightforward from Definition 2.4.

Proposition 2.9 (Subnormal backward extension of a 2-variable weighted shift). Consider the following 2-variable weighted shift (see Figure 2), and let $\mathcal{M}$ be the subspace associated to indices $\mathbf{k}$ with $k_{2} \geq 1$. Assume that $\mathbf{T}_{\mathcal{M}}:=\left.\mathbf{T}\right|_{\mathcal{M}}$ is subnormal with Berger measure $\mu_{\mathcal{M}}$ and that $W_{0}:=\operatorname{shift}\left(\alpha_{00}, \alpha_{10}, \cdots\right)$ is subnormal 


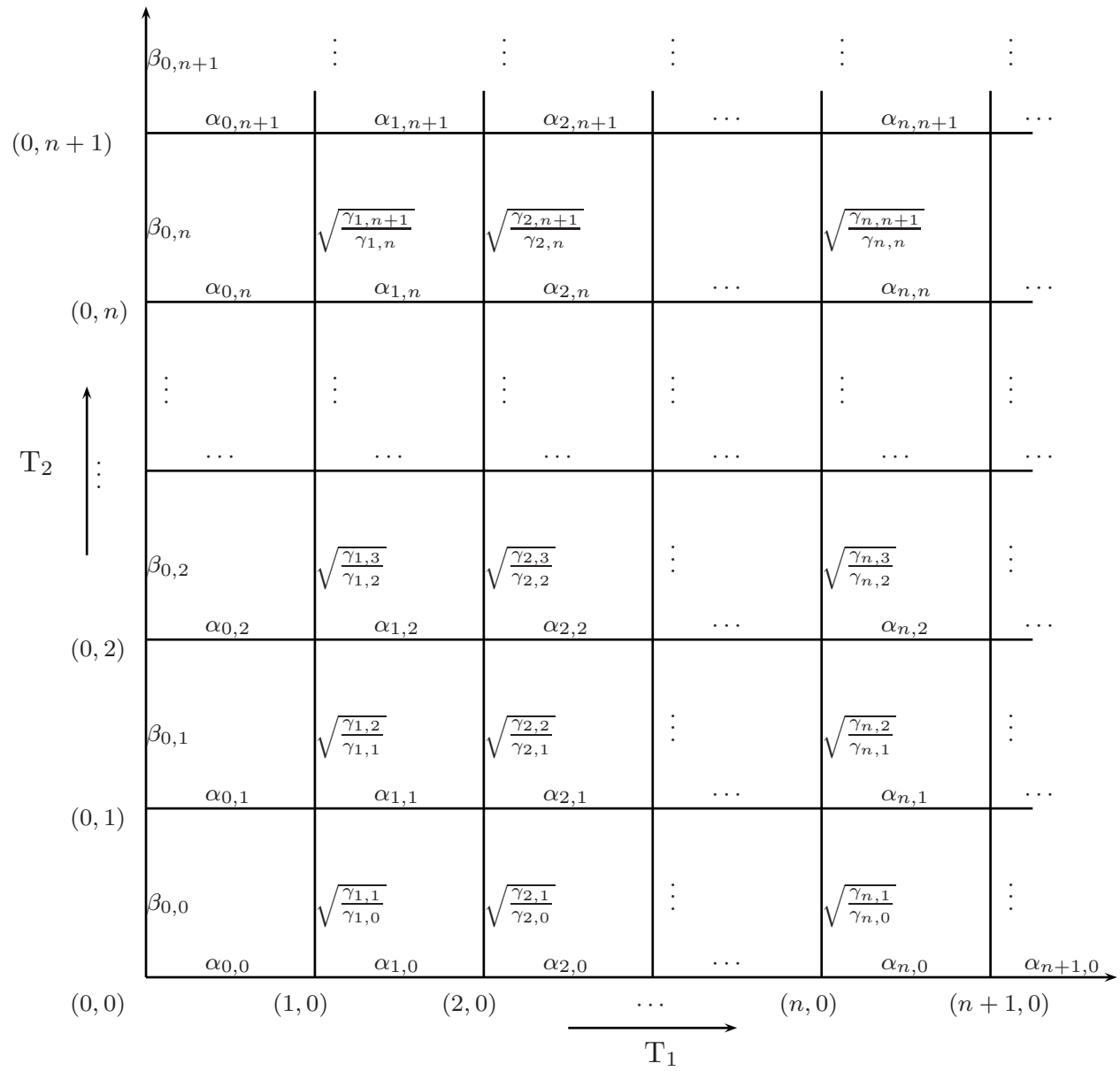

Figure 2. Weight diagram of the 2-variable weighted shift in Proposition 2.9

with Berger measure $\nu$. Then $\mathbf{T}$ is subnormal if and only if

(i) $\frac{1}{t} \in L^{1}\left(\mu_{\mathcal{M}}\right)$;

(ii) $\beta_{00}^{2} \leq\left(\left\|\frac{1}{t}\right\|_{L^{1}\left(\mu_{\mathcal{M}}\right)}\right)^{-1}$;

(iii) $\beta_{00}^{2}\left\|\frac{1}{t}\right\|_{L^{1}\left(\mu_{\mathcal{M}}\right)}\left(\mu_{\mathcal{M}}\right)_{e x t}^{X} \leq \nu$.

Moreover, if $\beta_{00}^{2}\left\|\frac{1}{t}\right\|_{L^{1}\left(\mu_{\mathcal{M}}\right)}=1$, then $\left(\mu_{\mathcal{M}}\right)_{\text {ext }}^{X}=\nu$. In the case when $\mathbf{T}$ is subnormal, the Berger measure $\mu$ of $\mathbf{T}$ is given by

$$
\begin{aligned}
d \mu(s, t)= & \beta_{00}^{2}\left\|\frac{1}{t}\right\|_{L^{1}\left(\mu_{\mathcal{M}}\right)} d\left(\mu_{\mathcal{M}}\right)_{e x t}(s, t) \\
& +\left(d \nu(s)-\beta_{00}^{2}\left\|\frac{1}{t}\right\|_{L^{1}\left(\mu_{\mathcal{M}}\right)} d\left(\mu_{\mathcal{M}}\right)_{e x t}^{X}(s)\right) d \delta_{0}(t) .
\end{aligned}
$$

Proof. $(\Rightarrow)$ First, observe that the moments of $\mathbf{T}$ and $\mathbf{T}_{\mathcal{M}}$ are related as follows:

$$
\gamma_{\mathbf{k}+\varepsilon_{2}}(\mathbf{T})=\beta_{00}^{2} \gamma_{\mathbf{k}}\left(\mathbf{T}_{\mathcal{M}}\right)\left(\text { all } \mathbf{k} \in \mathbb{Z}_{+}^{2}\right),
$$


so under the assumption that $\mathbf{T}$ is subnormal we must have

$$
\begin{aligned}
\iint s^{i} t^{j}(t d \mu)(s, t) & =\iint s^{i} t^{j+1} d \mu(s, t)=\gamma_{i, j+1}(\mathbf{T}) \\
& =\beta_{00}^{2} \gamma_{i j}\left(\mathbf{T}_{\mathcal{M}}\right)=\beta_{00}^{2} \iint s^{i} t^{j} d \mu_{\mathcal{M}}(s, t) .
\end{aligned}
$$

Thus $t d \mu(s, t)=\beta_{00}^{2} d \mu_{\mathcal{M}}(s, t)$ and $\mu_{\mathcal{M}}(E \times\{0\})=0$ for all $E \subseteq X$. It follows at once that

$$
\begin{aligned}
\iint \frac{1}{t} d \mu_{\mathcal{M}}(s, t) & =\iint_{(t>0)} \frac{1}{t} d \mu_{\mathcal{M}}(s, t)=\frac{1}{\beta_{00}^{2}} \iint_{(t>0)} \frac{1}{t} t d \mu(s, t) \\
& =\frac{1}{\beta_{00}^{2}} \mu((t>0)) \leq \frac{1}{\beta_{00}^{2}}
\end{aligned}
$$

which establishes parts (i) and (ii). As for part (iii), let $E \subseteq X$ and $F \subseteq Y$ be two arbitrary Borel sets. Then

$$
\begin{aligned}
\beta_{00}^{2} \| & \frac{1}{t} \|_{L^{1}\left(\mu_{\mathcal{M}}\right)}\left(\mu_{\mathcal{M})_{e x t}}(E \times F)\right. \\
& =\beta_{00}^{2}\left\|\frac{1}{t}\right\|_{L^{1}\left(\mu_{\mathcal{M}}\right)} \iint_{E \times F}\left(1-\delta_{0}(t)\right) \frac{1}{t\left\|\frac{1}{t}\right\|_{L^{1}\left(\mu_{\mathcal{M}}\right)}} d \mu_{\mathcal{M}}(s, t) \\
& =\iint_{E \times(F \backslash\{0\})} \frac{1}{t} \beta_{00}^{2} d \mu_{\mathcal{M}}(s, t)=\mu(E \times(F \backslash\{0\})) \\
& \leq \mu(E \times F),
\end{aligned}
$$

and by Lemmas 2.8 and 2.5. $\beta_{00}^{2}\left\|\frac{1}{t}\right\|_{L^{1}\left(\mu_{\mathcal{M}}\right)}\left(\mu_{\mathcal{M}}\right)_{e x t}^{X} \leq \mu^{X}=\nu$. Finally, observe that when $\beta_{00}^{2}\left\|\frac{1}{t}\right\|_{L^{1}\left(\mu_{\mathcal{M}}\right)}=1$, the inequality in (2.2) becomes an equality, and therefore $\left(\mu_{\mathcal{M}}\right)_{\text {ext }}^{X}=\nu$.

$(\Leftarrow)$ Assume that (i), (ii) and (iii) hold, and let

$$
\mu:=\beta_{00}^{2}\left\|\frac{1}{t}\right\|_{L^{1}\left(\mu_{\mathcal{M}}\right)}\left(\mu_{\mathcal{M}}\right)_{e x t}+\left[\nu-\beta_{00}^{2}\left\|\frac{1}{t}\right\|_{L^{1}\left(\mu_{\mathcal{M}}\right)}\left(\mu_{\mathcal{M}}\right)_{e x t}^{X}\right] \times \delta_{0} .
$$

Of course, if $\beta_{00}^{2}\left\|\frac{1}{t}\right\|_{L^{1}\left(\mu_{\mathcal{M}}\right)}=1$, then $\mu:=\left(\mu_{\mathcal{M}}\right)_{\text {ext }}$, since the total mass of the second summand is zero. We now compute the moments of $\mu$ and verify that they agree with the moments of $\mathbf{T}$. If $j>0$, then

$$
\begin{aligned}
\iint s^{i} t^{j} d \mu(s, t) & =\beta_{00}^{2}\left\|\frac{1}{t}\right\|_{L^{1}\left(\mu_{\mathcal{M}}\right)} \iint s^{i} t^{j} d\left(\mu_{\mathcal{M}}\right)_{e x t}(s, t) \\
& =\beta_{00}^{2}\left\|\frac{1}{t}\right\|_{L^{1}\left(\mu_{\mathcal{M}}\right)} \iint s^{i} t^{j}\left(1-\delta_{0}(t)\right) \frac{1}{t\left\|\frac{1}{t}\right\|_{L^{1}\left(\mu_{\mathcal{M}}\right)}} d \mu_{\mathcal{M}}(s, t) \\
& =\beta_{00}^{2} \iint s^{i} t^{j-1} d \mu_{\mathcal{M}}(s, t)=\beta_{00}^{2} \gamma_{(i, j-1)}\left(\mathbf{T}_{\mathcal{M}}\right)=\gamma_{(i, j)}(\mathbf{T}),
\end{aligned}
$$


as desired. When $j=0$, we have

$$
\begin{aligned}
\iint s^{i} d \mu(s, t)= & \beta_{00}^{2}\left\|\frac{1}{t}\right\|_{L^{1}\left(\mu_{\mathcal{M}}\right)} \iint s^{i} d\left(\mu_{\mathcal{M})_{e x t}}(s, t)\right. \\
& +\int s^{i} d\left(\nu-\beta_{00}^{2}\left\|\frac{1}{t}\right\|_{L^{1}\left(\mu_{\mathcal{M}}\right)}\left(\mu_{\mathcal{M}}\right)_{\text {ext }}^{X}\right)(s) \\
= & \beta_{00}^{2}\left\|\frac{1}{t}\right\|_{L^{1}\left(\mu_{\mathcal{M}}\right)} \int s^{i} d\left(\mu_{\mathcal{M}}\right)_{\text {ext }}^{X}(s) \\
& +\int s^{i} d \nu(s)-\beta_{00}^{2}\left\|\frac{1}{t}\right\|_{L^{1}\left(\mu_{\mathcal{M}}\right)} \int s^{i} d\left(\mu_{\mathcal{M}}\right)_{\text {ext }}^{X}(s) \\
& (\operatorname{using} \operatorname{Lemma} 2.5 \text { for the first term }) \\
= & \int s^{i} d \nu(s)=\gamma_{(i, 0)}(\mathbf{T}),
\end{aligned}
$$

as desired. It follows that $\mathbf{T}$ is subnormal, with Berger measure $\mu$.

We are now ready to exhibit our first family of counterexamples to Conjecture 1.4. Consider the 2-variable weighted shift given by Figure 3, where $\max \{x, y\}<1$ and $a<x$.

Proposition 2.10. The 2-variable weighted shift $\mathbf{T}$ given by Figure 3 is hyponormal if and only if $y \leq x \sqrt{\frac{1-x^{2}}{x^{2}-2 a^{2} x^{2}+a^{4}}}$.

Proof. By the Six-point Test (Theorem 1.3), to show the joint hyponormality of $\mathbf{T}$ it is enough to check that

$$
H:=\left(\begin{array}{cc}
1-x^{2} & \frac{a^{2} y}{x}-y x \\
\frac{a^{2} y}{x}-y x & 1-y^{2}
\end{array}\right) \geq 0 .
$$

Since $x<1$, the positivity of $H$ is equivalent to $\operatorname{det} H \geq 0$, i.e.,

$$
\left(1-x^{2}\right)\left(1-y^{2}\right) \geq\left(\frac{a^{2} y}{x}-y x\right)^{2},
$$

which in turn is equivalent to $y \leq x \sqrt{\frac{1-x^{2}}{x^{2}-2 a^{2} x^{2}+a^{4}}}$ (observe that $x^{2}-2 a^{2} x^{2}+a^{4}=$ $\left.x^{2}\left(1-x^{2}\right)+\left(x^{2}-a^{2}\right)^{2}>0\right)$.

Proposition 2.11. The 2-variable weighted shift $\mathbf{T}$ given by Figure 3 is subnormal if and only if $y \leq \sqrt{\frac{1-x^{2}}{1-a^{2}}}$.

Proof. From Figure 3, it is obvious that $\mathbf{T}_{\mathcal{M}} \cong\left(I \otimes S_{a}, U_{+} \otimes I\right)$ (recall that $S_{a}:=$ $\operatorname{shift}(a, 1,1, \cdots)$ and $U_{+}$is the (unweighted) unilateral shift). By Lemma 2.1, $\mathbf{T}_{\mathcal{M}}$ 


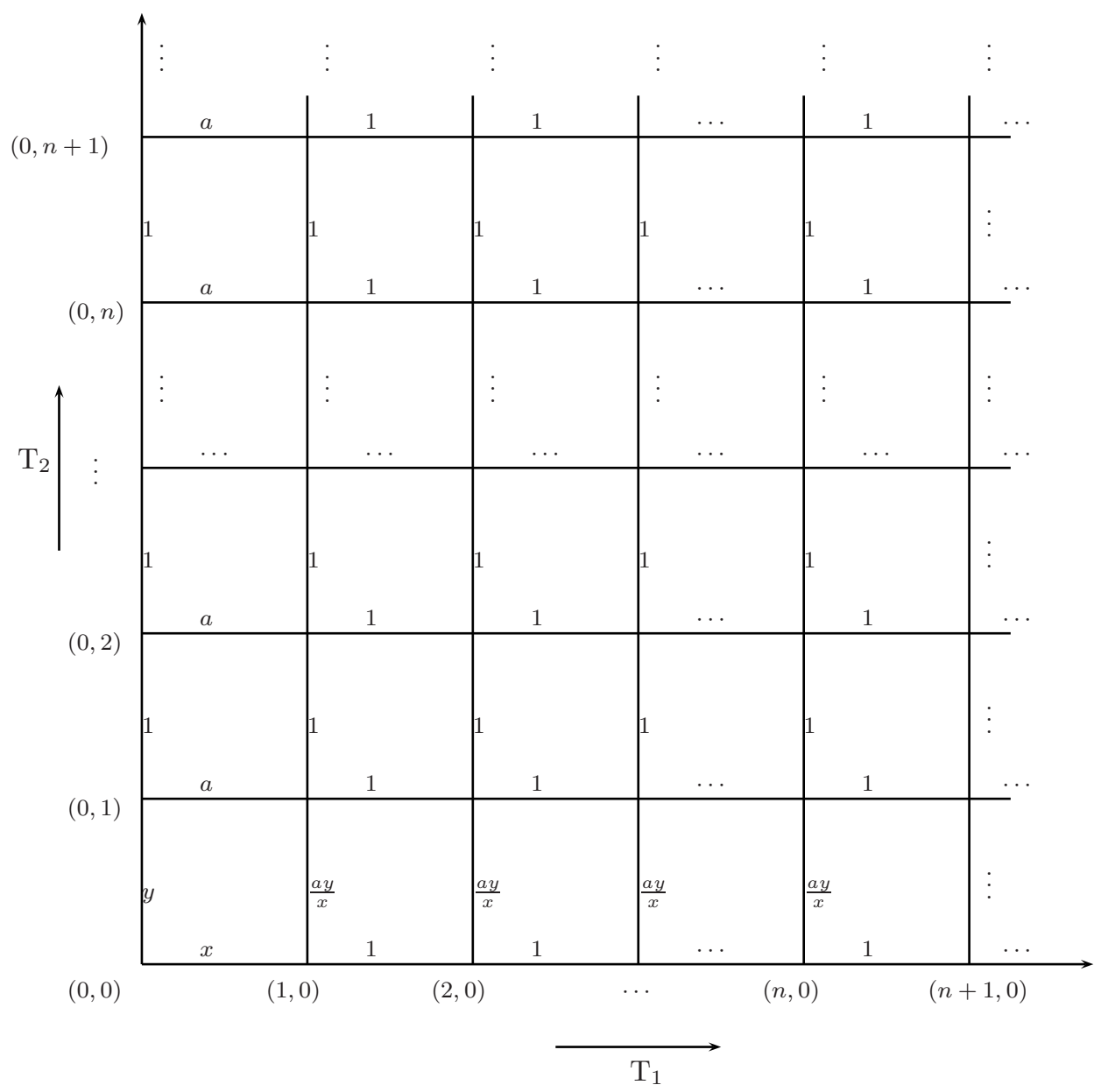

Figure 3. Weight diagram of the 2-variable weighted shift in Propositions 2.10 and 2.11

is subnormal, with Berger measure $\mu_{\mathcal{M}}:=\left[\left(1-a^{2}\right) \delta_{0}+a^{2} \delta_{1}\right] \times \delta_{1}$. By Proposition 2.9 ,

$$
\begin{aligned}
\mathbf{T} \text { is subnormal } & \Leftrightarrow \beta_{00}^{2}\left\|\frac{1}{t}\right\|_{L^{1}\left(\mu_{\mathcal{M}}\right)}\left(\mu_{\mathcal{M}}\right)_{e x t}^{X} \leq \nu \\
& \Leftrightarrow y^{2}\left[\left(1-a^{2}\right) \delta_{0}+a^{2} \delta_{1}\right] \leq\left(1-x^{2}\right) \delta_{0}+x^{2} \delta_{1} \\
& \Leftrightarrow y^{2}\left(1-a^{2}\right) \leq 1-x^{2} \text { and } a y \leq x \\
& \Leftrightarrow y \leq \min \left\{\frac{x}{a}, \sqrt{\frac{1-x^{2}}{1-a^{2}}}\right\} \\
& \Leftrightarrow y \leq \sqrt{\frac{1-x^{2}}{1-a^{2}}}(\text { since } \max \{x, y\}<1 \text { and } a<x) .
\end{aligned}
$$

We summarize the results in Propositions 2.10 and 2.11 as follows. 


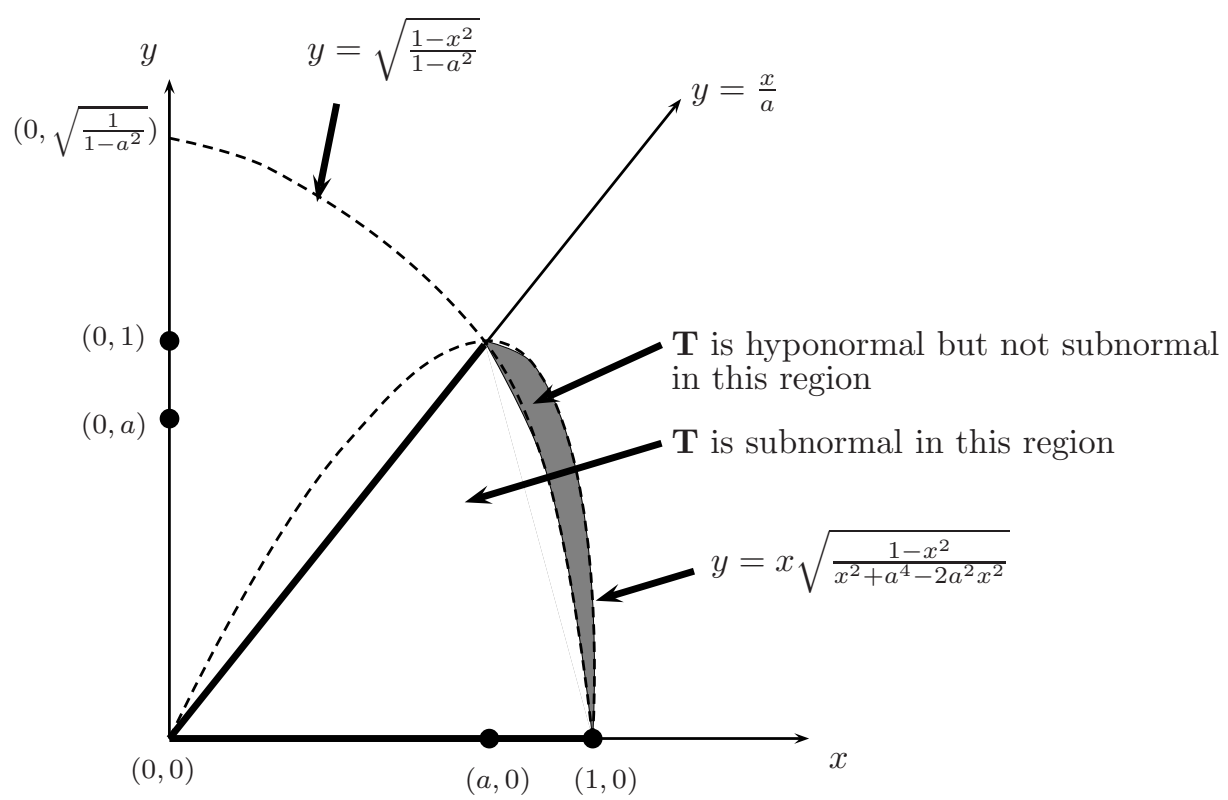

FigURE 4. Regions of hyponormality and subnormality for the 2variable weighted shift in Theorem 2.12

Theorem 2.12. The 2-variable weighted shift $\mathbf{T}$ given by Figure 3 is hyponormal and not subnormal if and only if $\sqrt{\frac{1-x^{2}}{1-a^{2}}}<y \leq x \sqrt{\frac{\left(1-x^{2}\right)}{x^{2}+a^{4}-2 a^{2} x^{2}}}$ (see Figure 4).

Remark 2.13. As exemplified in Figure 4, observe that for $x>a, \sqrt{\frac{1-x^{2}}{1-a^{2}}}<$ $x \sqrt{\frac{1-x^{2}}{x^{2}+a^{4}-2 a^{2} x^{2}}}<\frac{x}{a}$; for, if $a<x$ we have

$$
\begin{aligned}
a^{4} & <a^{2} x^{2} \Rightarrow x^{2}+a^{4}-2 a^{2} x^{2}<\left(1-a^{2}\right) x^{2} \\
& \Rightarrow \frac{1-x^{2}}{1-a^{2}}<\frac{x^{2}\left(1-x^{2}\right)}{x^{2}+a^{4}-2 a^{2} x^{2}}
\end{aligned}
$$

and

$$
\begin{aligned}
a^{2}\left(1-a^{2}\right) & <x^{2}\left(1-a^{2}\right) \Rightarrow a^{2}+a^{2} x^{2}<x^{2}+a^{4} \\
& \Rightarrow a^{2}\left(1-x^{2}\right)<x^{2}+a^{4}-2 a^{2} x^{2} \\
& \Rightarrow \frac{x^{2}\left(1-x^{2}\right)}{x^{2}+a^{4}-2 a^{2} x^{2}}<\frac{x^{2}}{a^{2}}
\end{aligned}
$$

as desired.

\section{The SECOND FAMily of COUntereXAMPles}

Construction of the family. Let $0<a, b<1$ and let $\left\{\xi_{k}\right\}_{k=0}^{\infty}$ and $\left\{\eta_{k}\right\}_{k=0}^{\infty}$ be two strictly increasing weight sequences. Consider the 2 -variable weighted shift $\mathbf{T} \equiv\left(T_{1}, T_{2}\right)$ on $\ell^{2}\left(\mathbb{Z}_{+}^{2}\right)$ given by the double-indexed weight sequences

$$
\alpha(\mathbf{k}):= \begin{cases}\xi_{k_{1}} & \text { if } k_{1} \geq 1 \text { or } k_{2} \geq 1 \\ a & \text { if } k_{1}=0 \text { and } k_{2}=0\end{cases}
$$




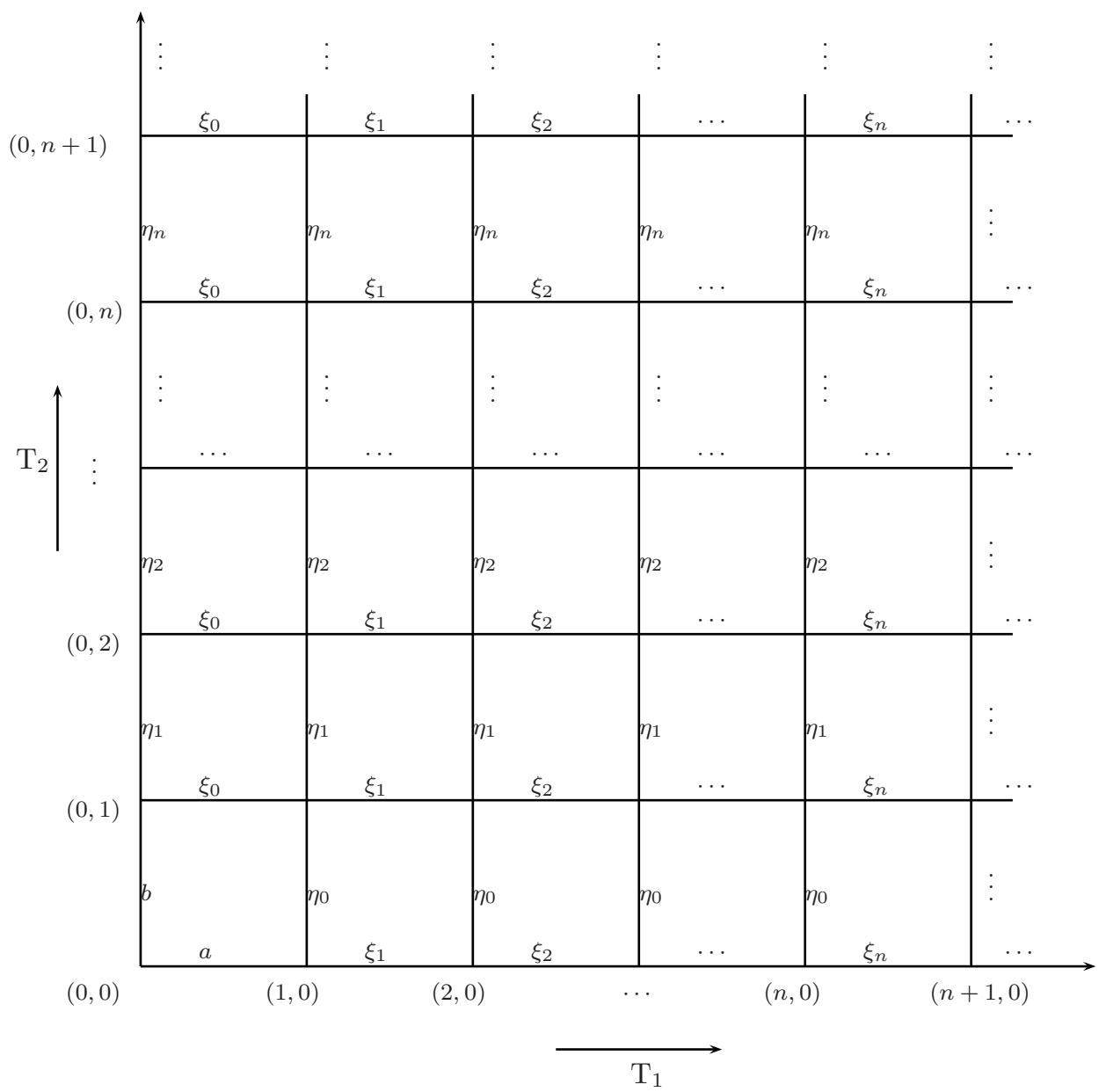

FiguRE 5 .

and

$$
\beta(\mathbf{k}):= \begin{cases}\eta_{k_{2}} & \text { if } k_{1} \geq 1 \text { or } k_{2} \geq 1 \\ b & \text { if } k_{1}=0 \text { and } k_{2}=0\end{cases}
$$

where $W_{\xi}$ and $W_{\eta}$ are two single-variable subnormal weighted shifts with Berger measures $\nu$ and $\omega$, resp., and

$$
a \eta_{0}=b \xi_{0}
$$

(to guarantee the commutativity of $T_{1}$ and $T_{2}$; cf. (1.1)). $\mathbf{T}$ can be represented by the following weight diagram (Figure 5). It is then clear that $T_{1}$ and $T_{2}$ are subnormal provided $a \leq \xi_{\text {ext }}\left(\nu_{\mathcal{M}}\right)$ and $b \leq \eta_{\text {ext }}\left(\omega_{\mathcal{M}}\right)$, where, as usual, $\mathcal{M}:=$ $\bigvee\left\{e_{1}, e_{2}, \cdots\right\}$; in particular, $a<\xi_{1}$ and $b<\eta_{1}$.

Proposition 3.1. The 2-variable weighted shift $\mathbf{T}$ defined by (3.1) and (3.2) is subnormal only if $a \leq s$, where $s:=\sqrt{\frac{\xi_{0}^{2} \xi_{1}^{2} \eta_{1}^{2}}{\xi_{1}^{2} \eta_{0}^{2}+\xi_{0}^{2} \eta_{1}^{2}-\xi_{0}^{2} \eta_{0}^{2}}}$. 
Proof. Suppose that $\mathbf{T}$ above is subnormal, and let $\mu$ be its Berger measure. Then the following partial moment matrix $M$, corresponding to the moments of $\mu$ associated with the monomials $1, s, t$ and $t s$, must be positive semi-definite:

$$
M:=\left(\begin{array}{cccc}
1 & a^{2} & b^{2} & a^{2} \eta_{0}^{2} \\
a^{2} & a^{2} \xi_{1}^{2} & a^{2} \eta_{0}^{2} & a^{2} \eta_{0}^{2} \xi_{1}^{2} \\
b^{2} & a^{2} \eta_{0}^{2} & b^{2} \eta_{1}^{2} & a^{2} \eta_{0}^{2} \eta_{1}^{2} \\
a^{2} \eta_{0}^{2} & a^{2} \eta_{0}^{2} \xi_{1}^{2} & a^{2} \eta_{0}^{2} \eta_{1}^{2} & a^{2} \eta_{0}^{2} \xi_{1}^{2} \eta_{1}^{2}
\end{array}\right) .
$$

Now, using Mathematica we obtain

$$
\begin{aligned}
\operatorname{det}(M) & \geq 0 \\
& \Leftrightarrow a^{6} \eta_{0}^{4}\left(\xi_{1}^{2}-\xi_{0}^{2}\right)\left(\eta_{1}^{2}-\eta_{0}^{2}\right)\left(a^{2} \xi_{0}^{2} \eta_{0}^{2}-a^{2} \xi_{1}^{2} \eta_{0}^{2}-a^{2} \xi_{0}^{2} \eta_{1}^{2}+\xi_{0}^{2} \xi_{1}^{2} \eta_{1}^{2}\right) \geq 0 \\
& \Leftrightarrow a^{2} \xi_{0}^{2} \eta_{0}^{2}-a^{2} \xi_{1}^{2} \eta_{0}^{2}-a^{2} \xi_{0}^{2} \eta_{1}^{2} \\
& \Leftrightarrow a \leq \xi_{0}^{2} \xi_{1}^{2} \eta_{1}^{2} \geq 0 \\
& \Leftrightarrow a \sqrt{\frac{\xi_{0}^{2} \xi_{1}^{2} \eta_{1}^{2}}{\xi_{1}^{2} \eta_{0}^{2}+\xi_{0}^{2} \eta_{1}^{2}-\xi_{0}^{2} \eta_{0}^{2}}}=s .
\end{aligned}
$$

Proposition 3.2. The 2-variable weighted shift $\mathbf{T}$ defined by (3.1) and (3.2) is hyponormal if and only if $a \leq h$, where $h:=\xi_{0} \sqrt{\frac{\xi_{1}^{2} \eta_{1}^{2}-\xi_{0}^{2} \eta_{0}^{2}}{\xi_{0}^{2} \eta_{1}^{2}+\xi_{1}^{2} \eta_{0}^{2}-2 \xi_{0}^{2} \eta_{0}^{2}}}$.

Proof. From the definition of $\mathbf{T}$ and the Six-point Test (Theorem 1.3), it is clear that all we need is for the following matrix to be positive semi-definite:

$$
L:=\left(\begin{array}{cc}
\xi_{1}^{2}-a^{2} & \xi_{0} \eta_{0}-a b \\
\xi_{0} \eta_{0}-a b & \eta_{1}^{2}-b^{2}
\end{array}\right)
$$

Observe that

$$
\begin{aligned}
\operatorname{det} L & \geq 0 \Leftrightarrow \xi_{1}^{2} \eta_{1}^{2}-\xi_{1}^{2} b^{2}-\xi_{0}^{2} \eta_{0}^{2}-a^{2} \eta_{1}^{2}+2 a b \xi_{0} \eta_{0} \geq 0 \\
& \Leftrightarrow \xi_{1}^{2} \eta_{1}^{2}-\xi_{1}^{2} \frac{a^{2} \eta_{0}^{2}}{\xi_{0}^{2}}-\xi_{0}^{2} \eta_{0}^{2}-a^{2} \eta_{1}^{2}+2 a^{2} \eta_{0}^{2} \geq 0 \quad\left(\text { using } b \xi_{0}=a \eta_{0} ; \text { cf. (3.3) }\right) \\
& \Leftrightarrow a^{2} \leq \frac{\xi_{0}^{2}\left(\xi_{1}^{2} \eta_{1}^{2}-\xi_{0}^{2} \eta_{0}^{2}\right)}{\xi_{0}^{2} \eta_{1}^{2}+\xi_{1}^{2} \eta_{0}^{2}-2 \xi_{0}^{2} \eta_{0}^{2}}=h^{2}
\end{aligned}
$$

(observe that $\xi_{0}^{2} \eta_{1}^{2}+\xi_{1}^{2} \eta_{0}^{2}-2 \xi_{0}^{2} \eta_{0}^{2}=\xi_{0}^{2}\left(\eta_{1}^{2}-\eta_{0}^{2}\right)+\left(\xi_{1}^{2}-\xi_{0}^{2}\right) \eta_{0}^{2}>0$, because the weight sequences are strictly increasing by hypothesis). Thus, $a \leq h$ is clearly a necessary condition for the hyponormality of $\mathbf{T}$. Now, a straightforward calculation shows that $h<\xi_{1}$; for,

$$
\xi_{1}^{2}-h^{2}=\frac{\eta_{0}^{2}\left(\xi_{1}^{2}-\xi_{0}^{2}\right)^{2}}{\xi_{0}^{2} \eta_{1}^{2}+\xi_{1}^{2} \eta_{0}^{2}-2 \xi_{0}^{2} \eta_{0}^{2}}>0 .
$$

It follows that $a \leq h$ implies $a<\xi_{1}$, and therefore $L \geq 0$ by the Nested Determinant Test Atk. Thus, the condition $a \leq h$ is also sufficient for the hyponormality of $\mathbf{T}$, and the proof is complete.

It follows from Propositions 3.1 and 3.2 that to ascertain the existence of a nonsubnormal, hyponormal 2-variable weighted shift $\mathbf{T}$ (with $T_{1}$ and $T_{2}$ subnormal), it suffices to show that for appropriate choices of $\xi_{0}, \xi_{1}, \eta_{0}$ and $\eta_{1}$, it is possible to obtain $s<h$, while keeping $a \leq \xi_{\text {ext }}\left(\nu_{\mathcal{M}}\right)$ and $b \equiv \frac{a \eta_{0}}{\xi_{0}} \leq \eta_{\text {ext }}\left(\omega_{\mathcal{M}}\right)$. Now,

$$
h^{2}-s^{2}=\frac{\xi_{0}^{4} \eta_{0}^{2}\left(\xi_{1}^{2}-\xi_{0}^{2}\right)\left(\eta_{1}^{2}-\eta_{0}^{2}\right)}{\left(\xi_{0}^{2} \eta_{1}^{2}+\xi_{1}^{2} \eta_{0}^{2}-2 \xi_{0}^{2} \eta_{0}^{2}\right)\left(\xi_{1}^{2} \eta_{0}^{2}+\xi_{0}^{2} \eta_{1}^{2}-\xi_{0}^{2} \eta_{0}^{2}\right)}>0 .
$$


Therefore, it suffices to prove the existence of strictly increasing weight sequences $\left\{\xi_{i}\right\}$ and $\left\{\eta_{j}\right\}$ such that

(i) $a \leq h$ (hyponormality of $\mathbf{T}$ ),

(ii) $a>s$ (nonsubnormality of $\mathbf{T}$ ),

(iii) $a \leq \xi_{\text {ext }}\left(\nu_{\mathcal{M}}\right)$ (subnormality of $T_{1}$ ),

(iv) $a \leq s_{2}:=\frac{\xi_{0}}{\eta_{0}} \eta_{\text {ext }}\left(\omega_{\mathcal{M}}\right)$ (subnormality of $T_{2}$ ).

We now seek to determine the relative positions of $h, s, s_{2}, \xi_{0}, \xi_{\text {ext }}\left(\nu_{\mathcal{M}}\right)$ and $\xi_{1}$ in the positive real axis.

Claim 1: $\xi_{0} \leq \xi_{\text {ext }}\left(\nu_{\mathcal{M}}\right)$. This follows from the fact that $\operatorname{shift}\left(\xi_{0}, \xi_{1}, \cdots\right)$ is subnormal.

Claim 2: $\xi_{0}<s$. For,

$$
s^{2}-\xi_{0}^{2}=\frac{\xi_{0}^{2} \xi_{1}^{2} \eta_{1}^{2}}{\xi_{1}^{2} \eta_{0}^{2}+\xi_{0}^{2} \eta_{1}^{2}-\xi_{0}^{2} \eta_{0}^{2}}-\xi_{0}^{2}=\frac{\xi_{0}^{2}\left(\xi_{1}^{2}-\xi_{0}^{2}\right)\left(\eta_{1}^{2}-\eta_{0}^{2}\right)}{\xi_{1}^{2} \eta_{0}^{2}+\xi_{0}^{2} \eta_{1}^{2}-\xi_{0}^{2} \eta_{0}^{2}}>0
$$

Claim 3: $h<\xi_{1}$. This was established in the proof of Proposition 3.2. cf. (3.4).

Claim 4: $h \leq s_{2}$ whenever

$$
\eta_{0} \leq u:=\frac{\xi_{1}^{2}\left(\eta_{1}^{2}-\eta_{e}^{2}\right)+2 \xi_{0}^{2} \eta_{e}^{2}-\sqrt{\left(\eta_{1}^{2}-\eta_{e}^{2}\right)\left(\xi_{1}^{4}\left(\eta_{1}^{2}-\eta_{e}^{2}\right)+4 \xi_{0}^{2} \eta_{e}^{2}\left(\xi_{1}^{2}-\xi_{0}^{2}\right)\right)}}{2 \xi_{0}^{2}} .
$$

Since

$$
s_{2}^{2}-h^{2}=\frac{\xi_{0}^{2}\left\{\xi_{0}^{2} \eta_{0}^{4}-\left[\xi_{1}^{2}\left(\eta_{1}^{2}-\eta_{e}^{2}\right)+2 \xi_{0}^{2} \eta_{e}^{2}\right] \eta_{0}^{2}+\xi_{0}^{2} \eta_{e}^{2} \eta_{1}^{2}\right\}}{\eta_{0}^{2}\left(\xi_{0}^{2} \eta_{1}^{2}+\xi_{1}^{2} \eta_{0}^{2}-2 \xi_{0}^{2} \eta_{0}^{2}\right)}
$$

it follows that $h \leq s_{2}$ if and only if the quadratic form

$$
\begin{aligned}
q(t) & \equiv A t^{2}+B t+C \\
& :=\xi_{0}^{2} t^{2}-\left[\xi_{1}^{2}\left(\eta_{1}^{2}-\eta_{e}^{2}\right)+2 \xi_{0}^{2} \eta_{e}^{2}\right] t+\xi_{0}^{2} \eta_{e}^{2} \eta_{1}^{2}
\end{aligned}
$$

is nonnegative. Since $A$ and $C$ are positive, and $B$ is negative, we need to study the discriminant, $\Delta:=B^{2}-4 A C$. Now,

$$
\begin{aligned}
\Delta & =\left(\xi_{1}^{2}\left(\eta_{1}^{2}-\eta_{e}^{2}\right)+2 \xi_{0}^{2} \eta_{e}^{2}\right)^{2}-4 \xi_{0}^{4} \eta_{e}^{2} \eta_{1}^{2} \\
& =\left(\eta_{1}^{2}-\eta_{e}^{2}\right)\left[\xi_{1}^{4} \eta_{1}^{2}-\eta_{e}^{2}\left(2 \xi_{0}^{2}-\xi_{1}^{2}\right)^{2}\right]
\end{aligned}
$$

so $\Delta \geq 0 \Leftrightarrow \xi_{1}^{4} \eta_{1}^{2}-\eta_{e}^{2}\left(2 \xi_{0}^{2}-\xi_{1}^{2}\right)^{2} \geq 0$. Since $\xi_{1}^{4} \eta_{1}^{2}-\eta_{e}^{2}\left(2 \xi_{0}^{2}-\xi_{1}^{2}\right)^{2}=\xi_{1}^{4}\left(\eta_{1}^{2}-\eta_{e}^{2}\right)$ $+4 \xi_{0}^{2} \eta_{e}^{2}\left(\xi_{1}^{2}-\xi_{0}^{2}\right)$, we see that $\Delta$ is always positive. We conclude that $q \geq 0$ on the interval $\left[0, t_{1}\right]$, where $t_{1}:=\frac{-B-\sqrt{\Delta}}{2 A}$ is the leftmost zero of $q$. Finally, a straightforward calculation shows that $t_{1}=u$.

We now summarize what we have so far. For $\eta_{0} \leq u$ we have

$$
\left\{\begin{array}{c}
\xi_{0}<s<h \leq s_{2}, \\
h<\xi_{1}, \\
\xi_{\text {ext }}\left(\nu_{\mathcal{M}}\right)<\xi_{1} \text { (by (1.5)). }
\end{array}\right.
$$

Thus, if we can ensure that $h \leq \xi_{\text {ext }}\left(\nu_{\mathcal{M}}\right)$, the construction of the example will be complete by taking $a$ such that $s<a \leq h$. Now, since $h \leq s_{2}$, an easy way to accomplish this is to build $\operatorname{shift}\left(\xi_{0}, \xi_{1}, \cdots\right)$ in such a way that $\xi_{\text {ext }}\left(\nu_{\mathcal{M}}\right)=s_{2}$. 
To do this, we appeal to Lemma 1.7 that is, we first build a 2-step recursively generated weighted shift whose first three weights are $s_{2}, \xi_{1}$ and $\xi_{2}$, and we then

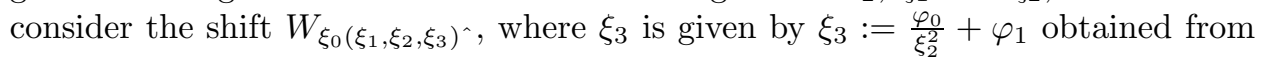
the equation $\gamma_{4}=\varphi_{0} \gamma_{2}+\varphi_{1} \gamma_{3}$. Observe that the extremal value of $W_{\left(\xi_{1}, \xi_{2}, \xi_{3}\right)^{\wedge}}$ is $s_{2}$, and that $\xi_{0}<s_{2}$, so the subnormality of $W_{\xi_{0}\left(\xi_{1}, \xi_{2}, \xi_{3}\right)^{\wedge}}$ is guaranteed. This completes the construction of the example.

Theorem 3.3. Let $\mathbf{T} \equiv\left(T_{1}, T_{2}\right)$ be the 2 -variable weighted shift defined by (3.1) and (3.2), and let

$$
\left\{\begin{aligned}
h & :=\xi_{0} \sqrt{\frac{\xi_{1}^{2} \eta_{1}^{2}-\xi_{0}^{2} \eta_{0}^{2}}{\xi_{0}^{2} \eta_{1}^{2}+\xi_{1}^{2} \eta_{0}^{2}-2 \xi_{0}^{2} \eta_{0}^{2}}} \\
s & :=\sqrt{\frac{\xi_{0}^{2} \xi_{1}^{2} \eta_{1}^{2}}{\xi_{1}^{2} \eta_{0}^{2}+\xi_{0}^{2} \eta_{1}^{2}-\xi_{0}^{2} \eta_{0}^{2}}}, \\
s_{2} & :=\frac{\xi_{0}}{\eta_{0}} \eta_{e}, \text { where } \eta_{e} \equiv \eta_{\text {ext }}\left(\omega_{\mathcal{M}}\right), \\
u & :=\frac{\xi_{0}^{2} \eta_{e}^{2} \eta_{1}^{2}}{\xi_{1}^{2}\left(\eta_{1}^{2}-\eta_{e}^{2}\right)+\xi_{0}^{2} \eta_{e}^{2}}, \text { and } \\
v & :=\frac{\xi_{1}^{2}\left(\eta_{1}^{2}-\eta_{e}^{2}\right)+2 \xi_{0}^{2} \eta_{e}^{2}-\sqrt{\left(\eta_{1}^{2}-\eta_{e}^{2}\right)\left(\xi_{1}^{4}\left(\eta_{1}^{2}-\eta_{e}^{2}\right)+4 \xi_{0}^{2} \eta_{e}^{2}\left(\xi_{1}^{2}-\xi_{0}^{2}\right)\right)}}{2 \xi_{0}^{2}}
\end{aligned}\right.
$$

Assume further that, as above, $s_{2}=\xi_{\text {ext }}\left(\nu_{\mathcal{M}}\right)$ and $\eta_{0} \leq \min \{u, v\}$. Finally, choose a such that $s<a \leq h$. Then

(i) $T_{1} T_{2}=T_{2} T_{1}$;

(ii) $T_{1}$ is subnormal;

(iii) $T_{2}$ is subnormal

(iv) $\mathbf{T}$ is hyponormal; and

(v) $\mathbf{T}$ is not subnormal.

Example 3.4. For a concrete numerical example, let $d \omega_{\mathcal{M}}(t):=2 d t$ on $\left[\frac{1}{2}, 1\right]$, so that $\left\|\frac{1}{t}\right\|_{L^{1}\left(\omega_{\mathcal{M}}\right)}=2 \ln 2$. It follows that $\eta_{e} \equiv \eta_{\text {ext }}\left(\omega_{\mathcal{M}}\right)=\frac{1}{\sqrt{2 \ln 2}}$ and $\eta_{1}=$ $\frac{\sqrt{3}}{2}$. Now take $\xi_{0}:=\frac{1}{2}$ and $\xi_{1}:=1$. Then $u=\frac{1}{4(2 \ln 2-1)} \cong 0.647$ and $v=$ $\frac{1}{4} \frac{6 \ln 2-2-\sqrt{2} \sqrt{(3 \ln 2-2)} \sqrt{(6 \ln 2-1)}}{\ln 2} \cong 0.523$, so we can take $\eta_{0}:=\frac{1}{2}$. With this choice of $\eta_{0}$ we obtain $s=\frac{\sqrt{2}}{2} \cong 0.707, h=\frac{1}{2} \sqrt{\frac{11}{5}} \cong 0.742$ and $s_{2}=\eta_{e}=\frac{1}{\sqrt{2 \ln 2}} \cong 0.849$. We can then take $a \in(s, h]$, for instance $a:=0.72$. To build the weighted shift $W_{\xi}$ we start with $s_{2}, \xi_{1}$ and $\xi_{2}:=\sqrt{2}$ to obtain $\varphi_{0}=\frac{1}{1-2 \ln 2}$ and $\varphi_{1}=\frac{1-4 \ln 2}{1-2 \ln 2}$. This gives $\xi_{3}=\frac{1}{2} \sqrt{\frac{16 \ln 2-5}{2 \ln 2-1}} \cong 1.985$. The 2-atomic measure $\nu_{\mathcal{M}}$ for $W_{\left(\xi_{1}, \xi_{2}, \xi_{3}\right)^{\text {}}}$. has atoms $t_{0} \cong 0.659$ and $t_{1} \cong 3.93$, and densities $\rho_{0} \cong 0.981$ and $\rho_{1} \cong 0.019$. With these values we can compute $\left\|\frac{1}{t}\right\|_{L^{1}\left(\nu_{\mathcal{M}}\right)} \cong 1.494$; observe that $\xi_{0}=\frac{1}{2} \leq$

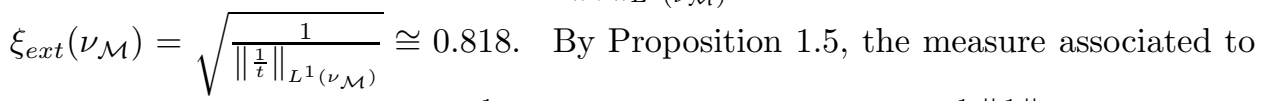
$\operatorname{shift}\left(\xi_{0}, \xi_{1}, \xi_{2}, \cdots\right)$ is $d \nu(t)=\frac{1}{4 t}\left(\rho_{0} d \delta_{t_{0}}(t)+\rho_{1} d \delta_{t_{1}}(t)\right)+\left(1-\frac{1}{4}\left\|\frac{1}{t}\right\|_{L^{1}(\nu \mathcal{M})}\right) d \delta_{0}(t)$. 


\section{The THIRD FAMILY OF COUNTEREXAMPLES}

Construction of the family. Let us consider the following 2-variable weighted shift (see Figure 6), where

$$
\begin{aligned}
& \text { (i) } 0<\xi_{1}<\xi_{2}<\cdots<\xi_{n} \nearrow 1 \\
& \text { (ii) } W_{\xi}:=\operatorname{shift}\left(\xi_{1}, \xi_{2}, \cdots\right) \text { is subnormal with Berger measure } \nu \\
& \text { (iii) } \frac{1}{s^{2}} \in L^{1}(\nu) \text { (this implies that } \frac{1}{s} \in L^{1}(\nu) \text {, by Jensen's inequality); } \\
& \text { (iv) } \xi_{e} \equiv \xi_{\text {ext }}:=\left(\int \frac{1}{s} d \nu(s)\right)^{-1 / 2} \\
& \text { (v) } a \leq \frac{1}{\xi_{e}}\left(\int \frac{1}{s^{2}} d \nu(s)\right)^{-1 / 2} \\
& \text { (vi) } b \leq \xi_{e}^{2}\left(\text { this implies the condition } b<\xi_{e}\right) \text {; and } \\
& \text { (vii) } a^{2} \leq \frac{b^{2}+\xi_{e}^{2}}{2} \text {. }
\end{aligned}
$$

(Recall that $\xi_{e}$ is the maximum possible value for $\xi_{0}$ in Proposition 1.5.)

Observe that $T_{1} \cong T_{2}$ and that $T_{1} T_{2}=T_{2} T_{1}$. We claim that $T_{1}$ (and therefore $\left.T_{2}\right)$ is subnormal. For, the choice of $\xi_{e}$ immediately implies that $\operatorname{shift}\left(\xi_{e}, \xi_{1}, \xi_{2}, \cdots\right)$ is subnormal, with Berger measure $d \nu_{e}(s):=\frac{\xi_{e}^{2}}{s} d \nu(s)$ (cf. Proposition 1.5). Another application of Proposition 1.5 shows that $\operatorname{shift}\left(a, \xi_{e}, \xi_{1}, \cdots\right)$ is subnormal if and only if $\frac{1}{s} \in L^{1}\left(\nu_{e}\right)$ (i.e., $\frac{1}{s^{2}} \in L^{1}(\nu)$, which is true by (4.1) (iii)) and $a^{2} \xi_{e}^{2} \int \frac{1}{s^{2}} d \nu(s)$ $\leq 1$, which holds by (4.1) $(\mathrm{v}))$. This implies that the restriction of $T_{1}$ to $\bigvee\left\{e_{(i, 0)}\right.$ : $i \geq 0\}$ is subnormal. Moreover, the subnormality of $T_{1}$ when restricted to $\bigvee\left\{e_{(i, j)}: i \geq 0\right\}(j>0)$ requires that $b \leq \xi_{e}$, which holds by (4.1) (vi).

For a concrete numerical example, consider the probability measure $d \nu(s):=$ $3 s^{2} d s$ on the interval $[0,1]$. The measure $\nu$ corresponds to a subnormal weighted shift with weights $\xi_{1}=\sqrt{\frac{3}{4}}, \xi_{2}=\sqrt{\frac{4}{5}}, \xi_{3}=\sqrt{\frac{5}{6}}, \cdots$. Indeed, in this case $W_{\xi}$ is the restriction of the Bergman shift $B_{+}$to the invariant subspace $\mathcal{M}_{2}$ obtained by removing the first two basis vectors in the canonical orthonormal basis of $\ell^{2}\left(\mathbb{Z}_{+}\right)$. Clearly $\frac{1}{s^{2}} \in L^{1}(\nu)$ and $\int \frac{1}{s^{2}} d \nu(s)=3$; moreover, $\int \frac{1}{s} d \nu(s)=\frac{3}{2}$, so in this case $\xi_{e}=\sqrt{\frac{2}{3}}$. Choosing $a=\sqrt{\frac{1}{2}}$ and $b=\sqrt{\frac{1}{3}}$ we see that all conditions in (4.1) are satisfied (cf. Corollary 4.4).

Proposition 4.1. The 2-variable weighted shift $\mathbf{T}$ given by Figure 6 is hyponormal.

Proof. Since the restriction of $\mathbf{T}$ to $\bigvee\left\{e_{(i, j)}: i, j \geq 1\right\}$ is clearly subnormal (being unitarily equivalent to $\left(I \otimes W_{\xi}, W_{\xi} \otimes I\right)$, and since the weight diagram of $\mathbf{T}$ is symmetric with respect to the diagonal $j=i$, it suffices to apply the Six-point Test (Theorem 1.3) to $\mathbf{k}=(i, 0)$, with $i \geq 0$.

Case 1: $\mathbf{k}=(0,0)$. Here we have

$$
\begin{aligned}
\left(\begin{array}{cc}
\xi_{e}^{2}-a^{2} & b^{2}-a^{2} \\
b^{2}-a^{2} & \xi_{e}^{2}-a^{2}
\end{array}\right) & \geq 0 \Leftrightarrow\left(\xi_{e}^{2}-a^{2}\right)^{2} \geq\left(b^{2}-a^{2}\right)^{2} \\
& \Leftrightarrow \xi_{e}^{2}-a^{2} \geq\left|b^{2}-a^{2}\right| .
\end{aligned}
$$




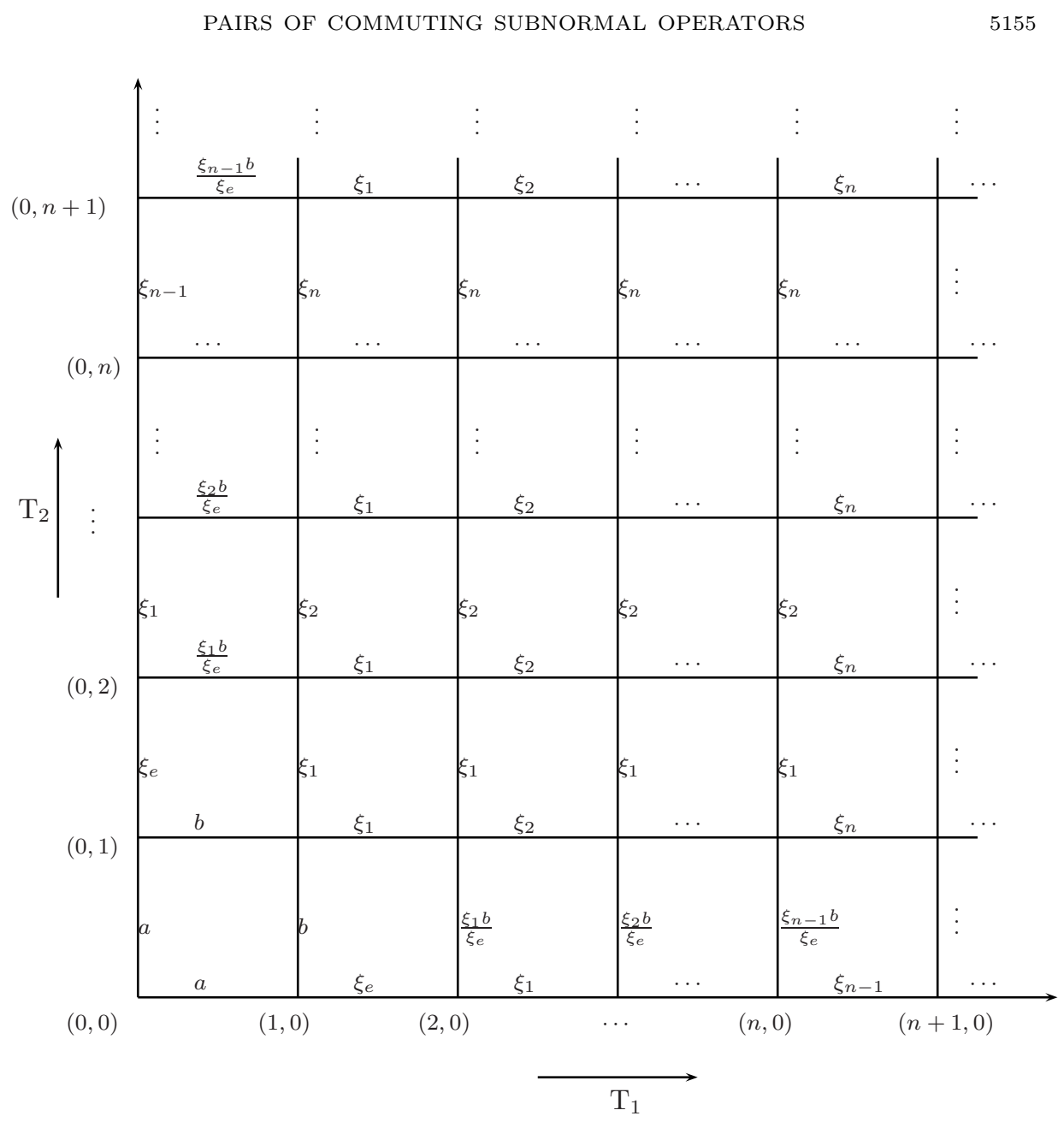

Figure 6 . Weight diagram of the 2-variable weighted shift in Example 19

When $b \leq a$, the last condition is equivalent to $2 a^{2} \leq b^{2}+\xi_{e}^{2}$, which holds by (4.1) (vii). When $b>a$, the condition is equivalent to $\xi_{e} \geq b$, which is guaranteed by (4.1) (vi).

Case 2: $\mathbf{k}=(1,0)$. Here

$$
\begin{aligned}
\left(\begin{array}{cc}
\xi_{1}^{2}-\xi_{e}^{2} & \frac{\xi_{1}^{2} b}{\xi_{e}}-b \xi_{e} \\
\frac{\xi_{1}^{2} b}{\xi_{e}}-b \xi_{e} & \xi_{1}^{2}-b^{2}
\end{array}\right) & \geq 0 \Leftrightarrow\left(\xi_{1}^{2}-\xi_{e}^{2}\right)\left(\xi_{1}^{2}-b^{2}\right) \geq\left(\frac{\xi_{1}^{2} b}{\xi_{e}}-b \xi_{e}\right)^{2} \\
& \Leftrightarrow \quad \xi_{1}^{2}-b^{2} \geq\left(\xi_{1}^{2}-\xi_{e}^{2}\right) \frac{b^{2}}{\xi_{e}^{2}} \Leftrightarrow b \leq \xi_{e}
\end{aligned}
$$

which again is guaranteed by (4.1) (vi). 
Case 3: $k=(n+1,0)(n \geq 1)$. Here

$$
\begin{aligned}
\left(\begin{array}{cc}
\xi_{n+1}^{2}-\xi_{n}^{2} & \frac{\xi_{n+1}^{2} b}{\xi_{e}}-\frac{\xi_{n}^{2} b}{\xi_{e}} \\
\frac{\xi_{n+1}^{2} b}{\xi_{e}}-\frac{\xi_{n}^{2} b}{\xi_{e}} & \xi_{1}^{2}-\frac{\xi_{n}^{2} b^{2}}{\xi_{e}^{2}}
\end{array}\right) & \geq 0 \\
& \Leftrightarrow\left(\xi_{n+1}^{2}-\xi_{n}^{2}\right)\left(\xi_{1}^{2}-\frac{\xi_{n}^{2} b^{2}}{\xi_{e}^{2}}\right) \geq\left(\frac{\xi_{n+1}^{2} b}{\xi_{e}}-\frac{\xi_{n}^{2} b}{\xi_{e}}\right)^{2} \\
& \Leftrightarrow \frac{\left(\xi_{n+1}^{2}-\xi_{n}^{2}\right)\left(\xi_{1}^{2} \xi_{e}^{2}-\xi_{n+1}^{2} b^{2}\right)}{\xi_{e}^{2}} \geq 0 \\
& \Leftrightarrow b \leq \frac{\xi_{1} \xi_{e}}{\xi_{n+1}}(\text { all } n \geq 1) .
\end{aligned}
$$

Since the sequence $\left\{\xi_{n}\right\}$ increases to 1 , the last inequality in (4.2) is equivalent to $b \leq \xi_{1} \xi_{e}$, which holds by (4.1) (vi).

The proof is now complete.

Proposition 4.2. The 2-variable weighted shift $\mathbf{T}$ given by Figure 6 is not subnormal if $p<0$, where $p:=\xi_{e}^{2} \xi_{1}^{4}+4 a^{2} b^{2} \xi_{1}^{2}-b^{2} \xi_{1}^{4}-a^{2} b^{2} \xi_{e}^{2}-a^{2} b^{4}-2 a^{2} \xi_{1}^{4}$.

Proof. Assume that $\mathbf{T}$ is subnormal, and consider the moment matrix associated to the monomials $1, x, y$ and $y x$ (cf. CuFi4, CuFi5), that is,

$$
M:=\left(\begin{array}{cccc}
1 & a^{2} & a^{2} & a^{2} b^{2} \\
a^{2} & a^{2} \xi_{e}^{2} & a^{2} b^{2} & a^{2} b^{2} \xi_{1}^{2} \\
a^{2} & a^{2} b^{2} & a^{2} \xi_{e}^{2} & a^{2} b^{2} \xi_{1}^{2} \\
a^{2} b^{2} & a^{2} b^{2} \xi_{1}^{2} & a^{2} b^{2} \xi_{1}^{2} & a^{2} b^{2} \xi_{1}^{4}
\end{array}\right) .
$$

In the presence of a representing measure, it is well known that $M$ must be positive semi-definite, so in particular $\operatorname{det} M \geq 0$. Now, a straightforward calculation shows that

$$
\begin{aligned}
\operatorname{det} M & =a^{6} b^{2}\left(\xi_{e}^{2}-b^{2}\right)\left(\xi_{e}^{2} \xi_{1}^{4}-\xi_{e}^{2} a^{2} b^{2}-2 a^{2} \xi_{1}^{4}-b^{2} \xi_{1}^{4}+4 a^{2} b^{2} \xi_{1}^{2}-b^{4} a^{2}\right) \\
& =a^{6} b^{2}\left(\xi_{e}^{2}-b^{2}\right) p .
\end{aligned}
$$

It follows that $p \geq 0$. Therefore, $\mathbf{T}$ is not subnormal whenever $p<0$, as desired.

Theorem 4.3. Let $a>0$ be such that $\sqrt{\frac{\xi_{e}^{2}}{2}}<a \leq \sqrt{\frac{\xi_{e}^{2}+\xi_{e}^{4}}{2}}$ and $a \leq \frac{1}{\xi_{e}}\left(\int \frac{1}{s^{2}} d \nu(s)\right)^{-1 / 2}$, and define $b:=\sqrt{2 a^{2}-\xi_{e}^{2}}$. Then the 2 -variable weighted shift $\mathbf{T} \equiv\left(T_{1}, T_{2}\right)$ satisfies (4.1)(i)-(vii), is hyponormal, and is not subnormal.

Proof. Observe that the condition $\sqrt{\frac{\xi_{e}^{2}}{2}}<a$ guarantees that $2 a^{2}>\xi_{e}^{2}$ (so $b$ is well defined) and that the condition $a \leq \sqrt{\frac{\xi_{e}^{2}+\xi_{e}^{4}}{2}}$ is equivalent to $2 a^{2}-\xi_{e}^{2} \leq \xi_{e}^{4}$ (so $b$ satisfies (4.1)(vi)). Moreover, $a^{2}=\frac{b^{2}+\xi_{e}^{2}}{2}$ trivially, so (4.1)(vii) also holds. It follows that $\mathbf{T}$ is hyponormal, by Proposition 4.1. To break subnormality, by Proposition 4.2 it suffices to show that $p$ is negative. Since $b^{2}=2 a^{2}-\xi_{e}^{2}$, we have

$$
\begin{aligned}
p= & \xi_{e}^{2} \xi_{1}^{4}-\xi_{e}^{2} a^{2}\left(2 a^{2}-\xi_{e}^{2}\right)-2 a^{2} \xi_{1}^{4}-\left(2 a^{2}-\xi_{e}^{2}\right) \xi_{1}^{4} \\
& +4 a^{2}\left(2 a^{2}-\xi_{e}^{2}\right) \xi_{1}^{2}-\left(2 a^{2}-\xi_{e}^{2}\right)^{2} a^{2} \\
= & -2\left(\xi_{1}^{2}-a^{2}\right)^{2}\left(2 a^{2}-\xi_{e}^{2}\right)<0,
\end{aligned}
$$

as desired. The proof is now complete. 
Corollary $4.4(\underline{\mathrm{DrMcC}})$. Let $d v(s):=3 s^{2} d s$ on $[0,1]$ and choose $a=\sqrt{\frac{1}{2}}$ and $b=\sqrt{\frac{1}{3}}$. Then the 2-variable weighted shift $\mathbf{T}$ given by Figure 6 is commuting, has subnormal components, is hyponormal, but is not subnormal.

Proof. By Theorem 4.3 and the comments preceding Proposition 4.1, it suffices to verify that $\sqrt{\frac{\xi_{e}^{2}}{2}}<a \leq \sqrt{\frac{\xi_{e}^{2}+\xi_{e}^{4}}{2}}$. Since $\xi_{e}=\sqrt{\frac{2}{3}}$ and $a=\sqrt{\frac{1}{2}}$, the result follows by a straightforward calculation.

\section{AN INSTANCE WHEN HYPONORMALITY SUFFICES}

In this section we will prove that under a suitable condition hyponormality does imply subnormality for commuting pairs of subnormal operators. We begin with an elementary result of independent interest.

Lemma 5.1. Let $\nu$ be a probability measure on $[0,1]$, and let $\gamma_{n} \equiv \gamma_{n}(\nu):=$ $\int s^{n} d \nu(s)(n \geq 0)$ be the moments of $\nu$. The sequence $\left\{\gamma_{n}\right\}_{n=0}^{\infty}$ is bounded below if and only if $\nu$ has an atom at $\{1\}$.

Proof. $(\Leftarrow)$ Let $\rho:=\nu(\{1\})>0$ and write $\nu \equiv(1-\rho) \eta+\rho \delta_{1}$, where $\eta$ is a probability measure on $[0,1]$ with $\eta(\{1\})=0$. It follows that $\gamma_{n}(\nu) \geq \rho \int s^{n} d \delta_{1}(s)=\rho$ (all $n \geq 0)$, so $\left\{\gamma_{n}\right\}$ is bounded below by $\rho$.

$(\Rightarrow)$ Suppose $\nu(\{1\})=0$, let $f_{n}(s):=s^{n}(0 \leq s \leq 1, n \geq 0)$, and consider the sequence of nonnegative functions $\left\{f_{n}\right\}_{n \geq 0}$. Clearly $f_{n} \searrow \chi_{\{1\}}$ pointwise, and $\left|f_{n}\right| \leq 1$ (all $n \geq 0$ ). By the Lebesgue Dominated Convergence Theorem, $\lim _{n \rightarrow \infty} \gamma_{n}=\lim _{n \rightarrow \infty} \int s^{n} d \nu(s)=\int \chi_{\{1\}} d \nu(s)=\nu(\{1\})=0$. Therefore, $\left\{\gamma_{n}\right\}$ is not bounded below.

We now consider the 2-variable weighted shift $\mathbf{T}$ given by Figure 7 , where $W_{\xi}:=$ $\operatorname{shift}\left(\xi_{0}, \xi_{1}, \cdots\right)$ is a subnormal contraction with associated measure $\nu$, and $y \leq 1$.

It is clear that $T_{1} T_{2}=T_{2} T_{1}$, and that $T_{1}$ is subnormal (being the orthogonal direct sum of $W_{\xi}$ and copies of $U_{+}$). To ensure the subnormality of $T_{2}$, we must impose the condition $\frac{y}{\sqrt{\gamma_{n}}} \leq 1$ (all $n \geq 0$ ), i.e., $y^{2} \leq \gamma_{n}$ (all $n \geq 0$ ), where $\gamma_{n} \equiv \gamma_{n}(\nu)$. Note that this condition also guarantees the boundedness of $\mathbf{T}$.

Theorem 5.2. Let $\mathbf{T}$ be the 2-variable weighted shift given by Figure 7 , and assume that $\mathbf{T}$ is hyponormal. Then $\mathbf{T}$ is subnormal.

Proof. We apply the Six-point Test (Theorem 1.3) to an arbitrary lattice point of the form $(n, 0)$. Since $\mathbf{T}$ is hyponormal by hypothesis, we must have

$$
\left(\xi_{n+1}^{2}-\xi_{n}^{2}\right)\left(1-\frac{y^{2}}{\gamma_{n}}\right) \geq\left(\frac{y}{\sqrt{\gamma_{n+1}}}-\frac{y \xi_{n}}{\sqrt{\gamma_{n}}}\right)^{2}
$$

or equivalently $\left(\xi_{n+1}^{2}-\xi_{n}^{2}\right)\left(1-\frac{y^{2}}{\gamma_{n}}\right) \geq \frac{y^{2}}{\gamma_{n}}\left(\frac{1}{\xi_{n}}-\xi_{n}\right)^{2}$, that is, $y^{2} \leq\left(\frac{\xi_{n+1}^{2}-\xi_{n}^{2}}{\xi_{n+1}^{2}+\frac{1}{\xi_{n}^{2}}-2}\right) \gamma_{n}$. Since $\xi_{n}^{2}+\frac{1}{\xi_{n}^{2}}-2 \geq 0$ and $\frac{\xi_{n+1}^{2}-\xi_{n}^{2}}{\xi_{n+1}^{2}+\frac{1}{\xi_{n}^{2}}-2}=\frac{\xi_{n+1}^{2}-\xi_{n}^{2}}{\left(\xi_{n+1}^{2}-\xi_{n}^{2}\right)+\xi_{n}^{2}+\frac{1}{\xi_{n}^{2}}-2}$, it follows that $\frac{\xi_{n+1}^{2}-\xi_{n}^{2}}{\xi_{n+1}^{2}+\frac{1}{\xi_{n}^{2}}-2}$ $\leq 1$, so $0<y^{2} \leq \gamma_{n}$ (all $n \geq 0$ ). Thus, $\left\{\gamma_{n}\right\}$ is bounded below, and by Lemma 5.1 we can write $\nu=(1-\rho) \eta+\rho \delta_{1}$, with $\rho:=\nu(\{1\})$ and $\eta(\{1\})=0$. It follows that $y^{2} \leq \rho$. Thus, $y^{2} \delta_{1} \leq \nu$. By Proposition 2.9, $\mathbf{T}$ is subnormal. 


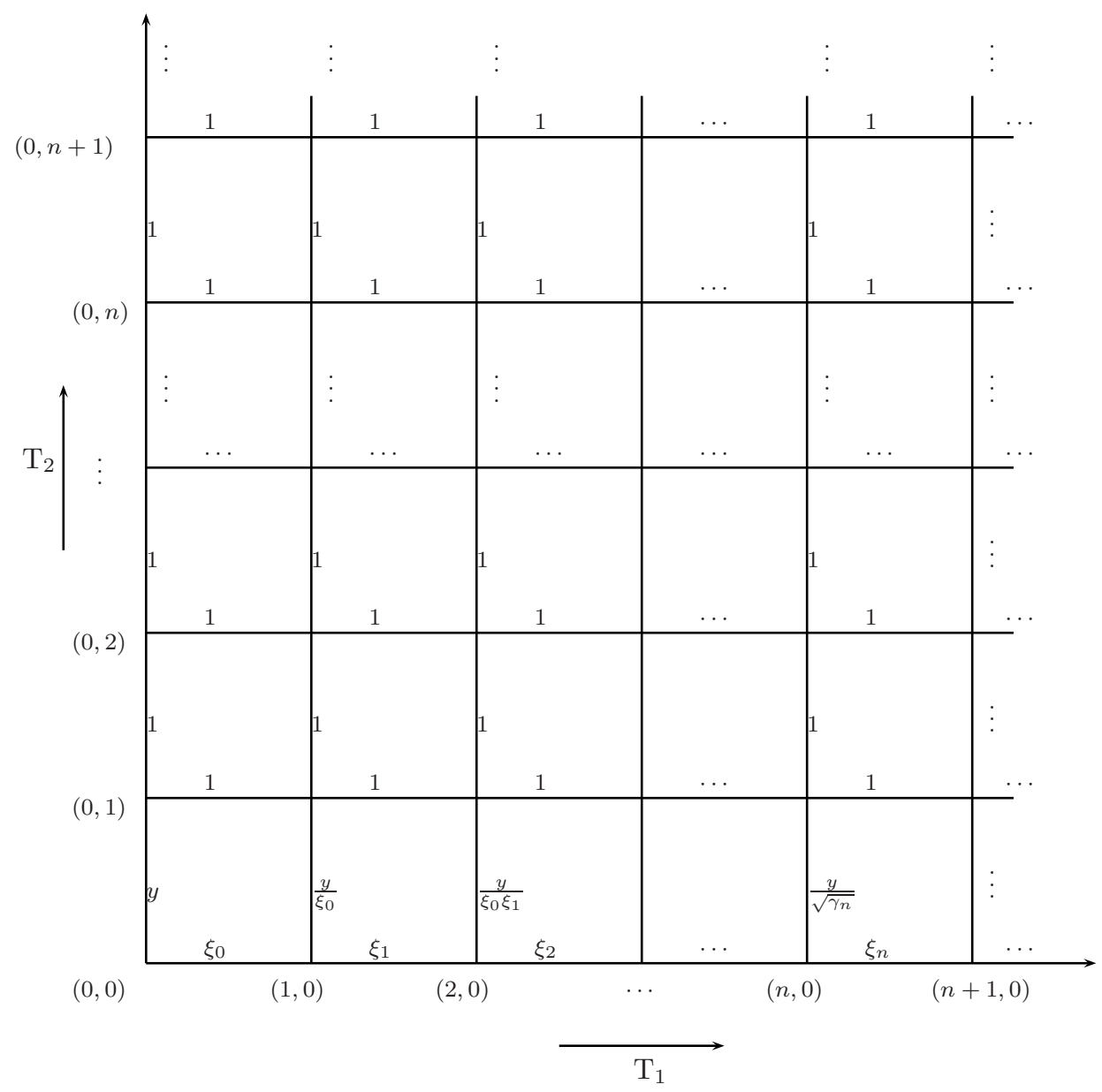

FiguRE 7. Weight diagram of the 2-variable weighted shift in Theorem 5.2

Remark 5.3. Theorem 5.2 (and its proof) reveals that for the 2-variable weighted shift given by Figure 7, the subnormality of $T_{2}$ is equivalent to the subnormality of $\mathbf{T}$, which in turn is equivalent to the hyponormality of $\mathbf{T}$.

\section{ACKNOWLEDGMENTS}

The authors are very grateful to the referee for several insightful comments about the mathematical content and presentation of the results in this article. All the examples and the basic construction in Section 3 were obtained using calculations with the software tool Mathematica [Wol].

\section{REFERENCES}

[Ath] A. Athavale, On joint hyponormality of operators, Proc. Amer. Math. Soc. 103(1988), 417-423. MR0943059 (89f:47033)

[Atk] K. Atkinson, Introduction to Numerical Analysis, Wiley and Sons, 2nd. Ed. 1989. MR:1007135 (90m:65001) 
[Con] J. Conway, The Theory of Subnormal Operators, Mathematical Surveys and Monographs, vol. 36, Amer. Math. Soc., Providence, 1991. MR.1112128(92h:47026)

[Cu1] R. Curto, Joint hyponormality: A bridge between hyponormality and subnormality, Proc. Symposia Pure Math. 51(1990), 69-91. MR.1077422 (91k:47049)

[Cu2] R. Curto, Quadratically hyponormal weighted shifts, Integral Equations Operator Theory 13(1990), 49-66. MR1025673 (90k:47061)

[Cu3] R. Curto, An operator-theoretic approach to truncated moment problems, in Linear Operators, Banach Center Publ., vol. 38, 1997, pp. 75-104. MR1457002 (99c:47014)

[CuFi1] R. Curto and L. Fialkow, Recursiveness, positivity, and truncated moment problems, Houston J. Math. 17(1991), 603-635. MR1147276 (93a:47016)

[CuFi2] R. Curto and L. Fialkow, Recursively generated weighted shifts and the subnormal completion problem, Integral Equations Operator Theory 17(1993), 202-246. MR.1233668 (94h:47050)

[CuFi3] R. Curto and L. Fialkow, Recursively generated weighted shifts and the subnormal completion problem, II, Integral Equations Operator Theory 18(1994), 369-426. MR.1265443 (94m:47044)

[CuFi4] R. Curto and L. Fialkow, Solution of the truncated complex moment problem with flat data, Memoirs Amer. Math. Soc. vol. 119, no. 568, Amer. Math. Soc., Providence, 1996. MR.1303090 (96g:47009)

[CuFi5] R. Curto and L. Fialkow, Solution of the singular quartic moment problem, J. Operator Theory 48(2002), 315-354. MR1938799 (2003j:47017)

[CuLe] R. Curto and W.Y. Lee, Towards a model theory for 2-hyponormal operators, Integral Equations Operator Theory 44(2002), 290-315. MR1933654 (2003m:47036)

[CMX] R. Curto, P. Muhly and J. Xia, Hyponormal pairs of commuting operators, Operator Theory: Adv. Appl. 35(1988), 1-22. MR.1017663 (90m:47037)

[DrMcC] M. Dritschel and S. McCullough, private communication.

[JeLu] N.P. Jewell and A.R. Lubin, Commuting weighted shifts and analytic function theory in several variables, J. Operator Theory 1(1979), 207-223. MR0532875 (80c:47030)

[Smu] Ju. L. Smul'jan, An operator Hellinger integral, Mat. Sb. (N.S.) 49 (1959), 381-430 (in Russian). MR.0121662 (22:12396)

[Sta] J. Stampfli, Which weighted shifts are subnormal?, Pacific J. Math. 17(1966), 367-379. MR 0193520 (33:1740)

[Wol] Wolfram Research, Inc. Mathematica, Version 4.2, Wolfram Research Inc., Champaign, IL, 2002 .

Department of Mathematics, The University of Iowa, Iowa City, Iowa 52242

E-mail address: rcurto@math.uiowa.edu

$U R L:$ http://www.math.uiowa.edu/ ${ }^{\sim}$ rcurto/

Department of Mathematics, Iowa State University, Ames, Iowa 50011

E-mail address: jyoon@iastate.edu

$U R L:$ http://www.public.iastate.edu/ jyoon/ 